Received by the editors: January 30, 2020; Accepted: July 11, 2020

\title{
ROBUST STABILITY ANALYSIS FOR FUZZY STOCHASTIC HOPFIELD NEURAL NETWORKS WITH TIME-VARYING DELAYS
}

\author{
N. GOPALAKRISHNAN
}

Department of Mathematics, Sri Ramakrishna Engineering College, Coimbatore - 641 022, Tamil Nadu, INDIA

\begin{abstract}
This paper investigates delay-dependent robust stability problem of fuzzy stochastic Hopfield neural networks with random time-varying delays. Moreover, in this paper, the stochastic delay is assumed to satisfy a certain probability distribution. By introducing a stochastic variable with Bernoulli distribution, the neural networks with random time delays is transformed into one with deterministic delays and stochastic parameters. Based on a Lyapunov-Krasovskii functional and stochastic analysis approach, delayprobability-distribution-dependent stability criteria have been derived in terms of linear matrix inequalities (LMIs), which can be checked easily by the LMI control toolbox. Finally two numerical examples are given to illustrate the effectiveness of the theoretical results.
\end{abstract}

\section{INTRODUCTION}

In recent decades, Neural Networks (NNs) especially recurrent neural networks (RNNs) and Hopfield neural networks (HNNs) have been successfully applied in various fields such as pattern recognition, optimization problems, associative memories, signal processing, etc., see [1] - 18. One of the best important works is to study the stability of the equilibrium point of NNs. Since time delays as a source of instability and poor performance always appear in many neural networks owing to the finite speed of information processing, the stability analysis for the delayed neural network has received considerable attention 347 .

2020 Mathematics Subject Classification. 92B20, 37H30, 15A39, 93C43.

Keywords and phrases. Hopfield neural networks, stochastic system, linear matrix inequality, time-varying delays

Submitted via ICCSPAM 2020.

gopalakrishnan.n@srec.ac.in

(D) 0000-0002-2365-9305.

(C)2020 Ankara University Communications Faculty of Sciences University of Ankara-Series A1 Mathematics and Statistics 
On the other hand, the stability analysis of stochastic systems with time delays has been investigated by many researchers since stochastic modelling plays an important role in many fields of science and engineering applications 8 8 - 16. In a real system, time delay often exists in a random form, that is, some values of the time delay are very large. However the probability of the delay taking such large values is very small and it may lead to a more conservative result, only if the information of variation range of the time delay is considered. In addition, its probabilistic characteristic such as Bernoulli distribution and the Poisson distribution can also be obtained by statistical methods. Therefore, it is necessary and realizable to investigate the probability-distribution delay and therefore in recent years, the stability problems of NNs with probability-distribution delay have been widely investigated 17,18 .

It is well known that fuzzy logic theory has shown to be an appealing and efficient approach to dealing with the analysis and synthesis problems for complex nonlinear systems. The well-known Takagi-Sugeno (T-S) fuzzy model [19], is a popular and convenient tool to transform a complex nonlinear system to a set of linear submodels via some fuzzy models by defining a linear input/output relationship as its consequence of individual plant rule. Recently, a lot of research works have been produced on T-S fuzzy model in the existing available literature $20-22]$.

Based on the above discussion, we consider the problem of delay-dependent robust stability analysis for uncertain fuzzy stochastic Hopfield neural networks with time-varying delays. Some sufficient condition for delay-probability-distributiondependent stability criteria of the addressed system have been derived in terms of linear matrix inequalities by constructing proper Lyapunov-Krasovskii functional and stochastic theory. Finally, numerical examples are provided to show the effectiveness of the theoretical results.

Notations: Throughout this paper, $\mathbb{R}^{n}$ and $\mathbb{R}^{n \times n}$ denote, respectively, the $\mathrm{n}$ dimensional Euclidean space and the set of all $n \times n$ real matrices. The superscript $T$ denotes the transposition and the notation $X \geq Y$ (respectively, $X>Y$ ), where $X$ and $Y$ are symmetric matrices, means that $X-Y$ is positive semi-definite (respectively, positive definite). $I_{n}$ is the $n \times n$ identity matrix. $\|\cdot\|$ is the Euclidean

norm in $\mathbb{R}^{n}$. Moreover, let $\left(\Omega, \mathcal{F},\left\{\mathcal{F}_{t}\right\}_{t \geq 0}, P\right)$ be a complete probability space with a filtration $\left\{\mathcal{F}_{t}\right\}_{t \geq 0}$ satisfying the usual conditions (i.e. the filtration contains all $P$-null sets and is right continuous). Denoted by $L_{\mathcal{F}_{0}}^{p}\left([-\bar{\tau}, 0] ; \mathbb{R}^{n}\right)$ the family of all $\mathcal{F}_{0}$-measurable $\mathcal{C}\left([-\bar{\tau}, 0] ; \mathbb{R}^{n}\right)$-valued random variables $\xi=\{\xi(\theta):-\bar{\tau} \leq \theta \leq 0\}$ such that $\sup _{-\bar{\tau} \leq \theta \leq 0} \mathbb{E}|\xi(\theta)|^{p}<\infty$, where $\mathbb{E}\{$.$\} stands for the mathematical expec-$ tation operator with respect to the given probability measure $P$.

\section{Problem description and preliminaries}

Consider the following uncertain stochastic HNNs with time-varying delays

$$
d x(t)=[-A(t) x(t)+B(t) f(x(t))+W(t) f(x(t-\tau(t)))] d t
$$




$$
\begin{aligned}
& +\left[H_{0}(t) x(t)+H_{1}(t) x(t-\tau(t))\right] d \omega(t), \\
x(t)= & \phi(t), \quad \forall t \in[-\bar{\tau}, 0],
\end{aligned}
$$

where $x(t) \in \mathbb{R}^{n}$ is the neural state vector, $f(x(t))=\left[f_{1}\left(x_{1}(t)\right), \ldots, f_{n}\left(x_{n}(t)\right)\right]^{T} \in$ $\mathbb{R}^{n}$ is the neuron activation function with initial condition $f(0)=0$. The timevarying delay $\tau(t)$ satisfies

$$
0 \leq \tau(t) \leq \bar{\tau}, \quad \dot{\tau}(t) \leq \mu
$$

where $\bar{\tau}$ and $\mu$ are constants. In (1), $A(t)=A+\Delta A(t), B(t)=B+\Delta B(t)$, $W(t)=W+\Delta W(t), H_{0}(t)=H_{0}+\Delta H_{0}(t)$ and $H_{1}(t)=H_{1}+\Delta H_{1}(t)$. Further $A=\operatorname{diag}\left\{a_{1}, a_{2}, \ldots, a_{n}\right\}$ has positive entries $a_{i}>0, B, W, H_{0}, H_{1}$ are connection weight matrices with appropriate dimensions and $\Delta A(t), \Delta B(t), \Delta W(t), \Delta H_{0}(t)$ and $\Delta H_{1}(t)$ denote the time-varying and norm-bounded uncertainties.

Assumption 2.1 The neuron activation function $f_{i}\left(x_{i}\right)$ satisfies

$$
0 \leq \frac{f_{i}\left(x_{i}\right)-f_{i}\left(y_{i}\right)}{x_{i}-y_{i}} \leq l_{i} \quad \forall x_{i}, y_{i} \in \mathbb{R}, \quad x_{i} \neq y_{i}, \quad i=1, \ldots, n
$$

Assumptions 2.2 Considering the information of probability distribution of the time delay $\tau(t)$, two sets and functions are defined by

$$
\begin{gathered}
\Omega_{1}=\left\{t: \tau(t) \in\left[0, \tau_{0}\right)\right\} \quad \text { and } \quad \Omega_{2}=\left\{t: \tau(t) \in\left[\tau_{0}, \bar{\tau}\right]\right\} \\
\tau_{1}(t)=\left\{\begin{array}{l}
\tau(t), \text { for } t \in \Omega_{1} \\
\bar{\tau}_{1}, \text { for } t \in \Omega_{2},
\end{array} \quad \text { and } \quad \tau_{2}(t)=\left\{\begin{array}{l}
\tau(t), \text { for } t \in \Omega_{2} \\
\bar{\tau}_{2}, \text { for } t \in \Omega_{1},
\end{array}\right.\right. \\
\dot{\tau}_{1}(t) \leq \mu_{1}<1, \quad \dot{\tau}_{2}(t) \leq \mu_{2}<1,
\end{gathered}
$$

where $\tau_{0} \in[0, \bar{\tau}], \bar{\tau}_{1} \in\left[0, \tau_{0}\right)$ and $\bar{\tau}_{2} \in\left[\tau_{0}, \bar{\tau}\right]$. It is easy to know $t \in \Omega_{1}$ means the event $\tau(t) \in\left[0, \tau_{0}\right)$ occurs and $t \in \Omega_{2}$ means the event $\tau(t) \in\left[\tau_{0}, \bar{\tau}\right]$ occurs. Therefore, a stochastic variable $\alpha(t)$ can be defined as

$$
\alpha(t)= \begin{cases}1, & \text { for } t \in \Omega_{1} \\ 0, & \text { for } t \in \Omega_{2}\end{cases}
$$

Assumption 2.3 $\alpha(t)$ is a Bernoulli distributed sequence with

$\operatorname{Prob}\{\alpha(t)=1\}=\mathbb{E}\{\alpha(t)\}=\alpha_{0}, \operatorname{Prob}\{\alpha(t)=0\}=1-\mathbb{E}\{\alpha(t)\}=1-\alpha_{0}$, where $0 \leq \alpha_{0} \leq 1$ is a constant and $\mathbb{E}\{\alpha(t)\}$ is the expectation of $\alpha(t)$.

Remark 2.4 From Assumption 2.3, it is easy to know that $\mathbb{E}\left\{\alpha(t)-\alpha_{0}\right\}=0, \mathbb{E}\left\{\left(\alpha(t)-\alpha_{0}\right)^{2}\right\}=\alpha_{0}\left(1-\alpha_{0}\right)$.

By Assumption 2.2 and 2.3, the system (1) can be rewritten as $d x(t)=\left[-A(t) x(t)+B(t) f(x(t))+\alpha(t) W(t) f\left(x\left(t-\tau_{1}(t)\right)\right)\right.$ 


$$
\begin{gathered}
\left.+(1-\alpha(t)) W(t) f\left(x\left(t-\tau_{2}(t)\right)\right)\right] d t \\
+\left[H_{0}(t) x(t)+\alpha(t) H_{1}(t) x\left(t-\tau_{1}(t)\right)+(1-\alpha(t)) H_{1}(t) x\left(t-\tau_{2}(t)\right)\right] d \omega(t), \\
x(t)=\xi(t), \quad t \in[-\bar{\tau}, 0],
\end{gathered}
$$

which is equivalent to

$$
\begin{aligned}
d x(t)= & {\left[-A(t) x(t)+B(t) f(x(t))+\alpha_{0} W(t) f\left(x\left(t-\tau_{1}(t)\right)\right)\right.} \\
& +\left(1-\alpha_{0}\right) W(t) f\left(x\left(t-\tau_{2}(t)\right)\right) \\
& \left.+\left(\alpha(t)-\alpha_{0}\right)\left(W(t) f\left(x\left(t-\tau_{1}(t)\right)\right)-W(t) f\left(x\left(t-\tau_{2}(t)\right)\right)\right)\right] d t \\
& +\left[H_{0}(t) x(t)+\alpha_{0} H_{1}(t) x\left(t-\tau_{1}(t)\right)+\left(1-\alpha_{0}\right) H_{1}(t) x\left(t-\tau_{2}(t)\right)\right. \\
& \left.+\left(\alpha(t)-\alpha_{0}\right)\left(H_{1}(t) x\left(t-\tau_{1}(t)\right)-H_{1}(t) x\left(t-\tau_{2}(t)\right)\right)\right] d \omega(t), \\
x(t)= & \xi(t), \quad t \in[-\bar{\tau}, 0] .
\end{aligned}
$$

Remark 2.5 In this paper, the probability distribution of the delay taking values in some interval is assumed to be known in advance. Further, a new model of the SNNs (8) has been derived, which can be seen as an extension of the common SNNs (1). Specially, in the case of $\alpha(t) \equiv 1$, system (8) becomes system (1). Moreover, when the probability of time delay taking values is known a priori, the possible values that the delay takes may be larger than those previously obtained results based on the traditional methods, which will be illustrated via example later.

In this paper, we consider the following neural network with parameter uncertainties and stochastic perturbations which is represented by a T-S fuzzy model. The $k$ th rule of the T-S fuzzy model is of the following form:

Plant Rule $k$ :

IF $\theta_{1}(t)$ is $\eta_{1}^{k}$ and $\ldots$ and $\theta_{p}(t)$ is $\eta_{p}^{k}$

THEN

$$
\begin{aligned}
d x(t)= & {\left[-A_{k}(t) x(t)+B_{k}(t) f(x(t))+\alpha_{0} W_{k}(t) f\left(x\left(t-\tau_{1}(t)\right)\right)\right.} \\
& +\left(1-\alpha_{0}\right) W_{k}(t) f\left(x\left(t-\tau_{2}(t)\right)\right) \\
& \left.+\left(\alpha(t)-\alpha_{0}\right)\left(W_{k}(t) f\left(x\left(t-\tau_{1}(t)\right)\right)-W_{k}(t) f\left(x\left(t-\tau_{2}(t)\right)\right)\right)\right] d t \\
& +\left[H_{0 k}(t) x(t)+\alpha_{0} H_{1 k}(t) x\left(t-\tau_{1}(t)\right)+\left(1-\alpha_{0}\right) H_{1 k}(t) x\left(t-\tau_{2}(t)\right)\right. \\
& \left.+\left(\alpha(t)-\alpha_{0}\right)\left(H_{1 k}(t) x\left(t-\tau_{1}(t)\right)-H_{1 k}(t) x\left(t-\tau_{2}(t)\right)\right)\right] d \omega(t) \\
x(t)= & \xi(t), \quad t \in[-\bar{\tau}, 0], \quad k=1,2, \ldots, r
\end{aligned}
$$


where $\eta_{i}^{k}(i=1,2, \ldots, p)$ is the fuzzy set, $\theta(t)=\left[\theta_{1}(t), \theta_{2}(t), \ldots, \theta_{p}(t)\right]^{T}$ is the premise variable vector and $r$ is the number of IF-THEN rules. $\omega(t)$ is a onedimensional Brownian motion defined on $\left(\Omega, \mathcal{F}_{t},\left\{\mathcal{F}_{t}\right\}_{t \geq 0}, P\right) . \xi \in L_{\mathcal{F}_{0}}^{2}\left([-\bar{\tau}, 0] ; \mathbb{R}^{n}\right)$ is the initial value of $(9) . A_{k}, B_{k}, W_{k}, H_{0 k}$ and $H_{1 k}$ are constant known real matrices. $\Delta A_{k}(t), \Delta B_{k}(t), \Delta W_{k}(t), \Delta H_{0 k}(t)$ and $\Delta H_{1 k}(t)$ denote the time-varying parameter uncertainties and we make the following assumption.

The parameter uncertainties $\Delta A_{k}(t), \Delta B_{k}(t), \Delta W_{k}(t), \Delta H_{0 k}(t)$ and $\Delta H_{1 k}(t)$ are of the form:

$$
\begin{gathered}
{\left[\begin{array}{ccccc}
\Delta A_{k}(t) & \Delta B_{k}(t) & \Delta W_{k}(t) & \Delta H_{0 k}(t) & \Delta H_{1 k}(t)
\end{array}\right]} \\
=G F(t)\left[\begin{array}{lllll}
E_{k}^{A} & E_{k}^{B} & E_{k}^{W} & E_{k}^{H_{0}} & E_{k}^{H_{1}}
\end{array}\right]
\end{gathered}
$$

where $G, E_{k}^{A}, E_{k}^{B}, E_{k}^{W}, E_{k}^{H_{0}}$ and $E_{k}^{H_{1}}$ are known real constant matrices with appropriate dimensions, and $F(t)$ is the time-varying uncertain matrix which satisfies

$$
F^{T}(t) F(t) \leq I
$$

The defuzzified output of the T-S fuzzy system (9) is represented as follows:

$$
\begin{aligned}
d x(t)= & \sum_{k=1}^{r} \mu_{k}(\theta(t))\left\{\left[-A_{k}(t) x(t)+B_{k}(t) f(x(t))+\alpha_{0} W_{k}(t) f\left(x\left(t-\tau_{1}(t)\right)\right)\right.\right. \\
& \quad+\left(1-\alpha_{0}\right) W_{k}(t) f\left(x\left(t-\tau_{2}(t)\right)\right) \\
+ & \left.\left(\alpha(t)-\alpha_{0}\right)\left(W_{k}(t) f\left(x\left(t-\tau_{1}(t)\right)\right)-W_{k}(t) f\left(x\left(t-\tau_{2}(t)\right)\right)\right)\right] d t \\
+ & {\left[H_{0 k}(t) x(t)+\alpha_{0} H_{1 k}(t) x\left(t-\tau_{1}(t)\right)+\left(1-\alpha_{0}\right) H_{1 k}(t) x\left(t-\tau_{2}(t)\right)\right.} \\
& \left.\left.+\left(\alpha(t)-\alpha_{0}\right)\left(H_{1 k}(t) x\left(t-\tau_{1}(t)\right)-H_{1 k}(t) x\left(t-\tau_{2}(t)\right)\right)\right] d \omega(t)\right\}
\end{aligned}
$$

where

$$
\mu_{k}(\theta(t))=\frac{v_{k}(\theta(t))}{\sum_{j=1}^{r} v_{j}(\theta(t))}, \quad v_{k}(\theta(t))=\prod_{j=1}^{p} \eta_{j}^{k}\left(\theta_{j}(t)\right)
$$

in which $\eta_{j}^{k}\left(\theta_{j}(t)\right)$ is the grade of membership of $\theta_{j}(t)$ in $\eta_{j}^{k}$. According to the theory of fuzzy sets, we have

$v_{k}(\theta(t)) \geq 0, \quad k=1,2, \ldots, r, \quad \sum_{k=1}^{r} v_{k}(\theta(t))>0$ for all $t$. Therefore, it implies

$\mu_{k}(\theta(t)) \geq 0, \quad k=1,2, \ldots, r, \quad \sum_{k=1}^{r} \mu_{k}(\theta(t))=1$ for all $t$.

Let $x(t ; \xi)$ denotes the state trajectory of system (12) from the initial value $x(\theta)=$ $\xi(\theta)$ on $-\bar{\tau} \leq \theta \leq 0$ in $L_{\mathcal{F}_{0}}^{2}\left([-\bar{\tau}, 0] ; \mathbb{R}^{n}\right)$. It is easy to see that system (12) admits a 
trivial solution $x(t ; 0) \equiv 0$.

The following definition and lemmas are used to prove our main result.

Definition 2.6 $22 \quad$ For system $(9)$ and every $\xi \in L_{\mathcal{F}_{0}}^{2}\left([-\infty, 0] ; \mathbb{R}^{n}\right)$, the trivial solution is asymptotically stable in the mean square if

$$
\lim _{t \rightarrow \infty} \mathbb{E}|x(t ; \xi)|^{2}=0 .
$$

Lemma 2.7 23 Let $D$ and $N$ be real constant matrices of appropriate dimensions, matrix $F(t)$ satisfies $F^{T}(t) F(t) \leq I$. Then (i) for any scalar $\epsilon>0$, $D F(t) N+N^{T} F^{T}(t) D^{T} \leq \epsilon^{-1} D D^{T}+\epsilon N^{T} N$.

(ii) For any $P>0,2 a^{T} b \leq a^{T} P^{-1} a+b^{T} P b$.

Lemma 2.8 24 For any constant matrix $M \in \mathbb{R}^{n \times n}, M=M^{T}>0$, scalar $\eta>0$, vector function $\omega:[0, \eta] \rightarrow \mathbb{R}^{n}$ such that the integrations are well defined, the following inequality holds

$$
\left(\int_{0}^{\eta} \omega(s) d s\right)^{T} M\left(\int_{0}^{\eta} \omega(s) d s\right) \leq \eta \int_{0}^{\eta} \omega^{T}(s) M \omega(s) d s .
$$

Lemma 2.9 25 Let $M, P, Q$ be the given matrices such that $Q>0$, then

$$
\left[\begin{array}{cc}
P & M^{T} \\
M & -Q
\end{array}\right]<0 \quad \Longleftrightarrow P+M^{T} Q^{-1} M<0
$$

Lemma 2.10 26] Let $U, V(t), W$ and $M$ be real matrices of appropriate dimension with $M$ satisfying $M=M^{T}$, then

$$
M+U V(t) W+W^{T} V^{T}(t) U^{T}<0 \text { forall } V^{T}(t) V(t) \leq I,
$$

if and only if there exists a scalar $\varepsilon>0$ such that

$$
M+\varepsilon^{-1} U U^{T}+\varepsilon W^{T} W<0 .
$$

Lemma 2.1127 Assume that $a(\cdot) \in \mathbb{R}^{n_{a}}, b(\cdot) \in \mathbb{R}^{n_{b}}$ and $N \in \mathbb{R}^{n_{a} \times n_{b}}$ are defined on the interval $\Omega$. Then for any matrices $X \in \mathbb{R}^{n_{a} \times n_{a}}, Y \in \mathbb{R}^{n_{a} \times n_{b}}$ and $Z \in \mathbb{R}^{n_{b} \times n_{b}}$, the following holds

$-2 \int_{\Omega} a^{T}(\alpha) N b(\alpha) d \alpha \leq \int_{\Omega}\left[\begin{array}{c}a(\alpha) \\ b(\alpha)\end{array}\right]^{T}\left[\begin{array}{cc}X & Y-N \\ & Z\end{array}\right]\left[\begin{array}{c}a(\alpha) \\ b(\alpha)\end{array}\right] d \alpha$, where $\left[\begin{array}{cc}X & Y \\ & Z\end{array}\right] \geq 0$.

\section{Main Results}

In this section, we consider a general stochastic system $d x(t)=f(x(t), t) d t+$ $g(x(t), t) d \omega(t)$ on $t \geq t_{0}$ with initial value $x\left(t_{0}\right)=x_{0} \in \mathbb{R}^{n}$, where $f: \mathbb{R}^{n} \times$ $\mathbb{R}^{+} \rightarrow \mathbb{R}^{n}$ and $g: \mathbb{R}^{n} \times \mathbb{R}^{+} \rightarrow \mathbb{R}^{n \times m}$. Let $\mathcal{C}^{2,1}\left(\mathbb{R}^{n} \times \mathbb{R}^{+} ; \mathbb{R}^{+}\right)$denotes the family of all nonnegative functions $V(x(t), t)$ on $\mathbb{R}^{n} \times \mathbb{R}^{+}$which are continuously twice differentiable in $x$ and once differentiable in $t$. Let $V \in \mathcal{C}^{2,1}\left(\mathbb{R}^{n} \times \mathbb{R}^{+} ; \mathbb{R}^{+}\right)$, an operator $\mathcal{L} V$ is defined from $\mathbb{R}^{n} \times \mathbb{R}^{+}$to $\mathbb{R}$ by

$$
\mathcal{L} V(x(t), t)=V_{t}(x(t), t)+V_{x}(x(t), t) f(x(t), t)
$$




$$
+\frac{1}{2} \operatorname{trace}\left[g^{T}(x(t), t) V_{x x}(x(t), t) g(x(t), t)\right]
$$

where

$$
\begin{aligned}
V_{t}(x(t), t) & =\frac{\partial V(x(t), t)}{\partial t}, V_{x}(x(t), t)=\left(\frac{\partial V(x(t), t)}{\partial x_{1}}, \ldots, \frac{\partial V(x(t), t)}{\partial x_{n}}\right), \\
V_{x x}(x(t), t) & =\left(\frac{\partial^{2} V(x(t), t)}{\partial x_{i} \partial x_{j}}\right)_{n \times n} .
\end{aligned}
$$

Then, by Ito's formula, one can have

$$
\mathbb{E} V(x(t), t)=\mathbb{E} V\left(x_{0}, t_{0}\right)+\mathbb{E} \int_{t_{0}}^{t} \mathcal{L} V(x(s), s) d s .
$$

Now, we define the following variables:

$$
\begin{aligned}
\bar{A}+\Delta \bar{A}(t)=\sum_{k=1}^{r} \mu_{k}(\theta(t))\left(A_{k}+\Delta A_{k}(t)\right), \bar{B}+\Delta \bar{B}(t)=\sum_{k=1}^{r} \mu_{k}(\theta(t))\left(B_{k}+\Delta B_{k}(t)\right), \\
\bar{W}+\Delta \bar{W}(t)=\sum_{k=1}^{r} \mu_{k}(\theta(t))\left(W_{k}+\Delta W_{k}(t)\right), \\
\bar{H}_{0}+\Delta \bar{H}_{0}(t)=\sum_{k=1}^{r} \mu_{k}(\theta(t))\left(H_{0 k}+\Delta H_{0 k}(t)\right), \\
\bar{H}_{1}+\Delta \bar{H}_{1}(t)=\sum_{k=1}^{r} \mu_{k}(\theta(t))\left(H_{1 k}+\Delta H_{1 k}(t)\right),
\end{aligned}
$$

by using the above notations and parameter uncertainties are not taken into account, then system (12) can be rewritten as

$$
\begin{aligned}
d x(t)= & {\left[-\bar{A} x(t)+\bar{B} f(x(t))+\alpha_{0} \bar{W} f\left(x\left(t-\tau_{1}(t)\right)\right)+\left(1-\alpha_{0}\right) \bar{W} f\left(x\left(t-\tau_{2}(t)\right)\right)\right.} \\
& \left.+\left(\alpha(t)-\alpha_{0}\right)\left(\bar{W} f\left(x\left(t-\tau_{1}(t)\right)\right)-\bar{W} f\left(x\left(t-\tau_{2}(t)\right)\right)\right)\right] d t \\
& +\left[\bar{H}_{0} x(t)+\alpha_{0} \bar{H}_{1} x\left(t-\tau_{1}(t)\right)+\left(1-\alpha_{0}\right) \bar{H}_{1} x\left(t-\tau_{2}(t)\right)\right. \\
& \left.+\left(\alpha(t)-\alpha_{0}\right)\left(\bar{H}_{1} x\left(t-\tau_{1}(t)\right)-\bar{H}_{1} x\left(t-\tau_{2}(t)\right)\right)\right] d \omega(t)
\end{aligned}
$$

Now, we discuss the stability criteria for stochastic neural network (14) without uncertainties as follows

Theorem 3.1 For given scalars $\bar{\tau}_{1}, \bar{\tau}_{2}, \mu_{1}, \mu_{2}$, and $0<\alpha_{0}<1$ satisfying $\alpha_{0} \mu_{1}<1$, the SNNs (14) is globally asymptotically stable in the mean square, if there exist symmetric positive definite matrices $P>0, Q_{i}>0(i=1,2,3,4,5,6)$, 
$R_{1}>0, R_{2}>0, Z_{1}>0$, and $Z_{2}>0$, for any matrices $X, Y$ and positive diagonal matrices $K_{1}>0, K_{2}>0$ and $K_{3}>0$ such that the following LMIs

$$
\left[\begin{array}{ll}
X & Y \\
& Z_{1}
\end{array}\right] \geq 0 \text { and }\left[\begin{array}{ll}
X & Y \\
& Z_{2}
\end{array}\right] \geq 0
$$

$\Upsilon_{k}=\left[\begin{array}{ccccccccc}\Omega_{k} & \bar{\tau}_{1} \eta_{1} Z_{1} & \sigma \bar{\tau}_{1} \eta_{2} Z_{1} & \bar{\tau}_{2} \eta_{1} Z_{2} & \sigma \bar{\tau}_{2} \eta_{2} Z_{2} & \vartheta_{1} \bar{P} & \sigma \vartheta_{2} \bar{P} & Y & Y \\ & -\bar{\tau}_{1} Z_{1} & 0 & 0 & 0 & 0 & 0 & 0 & 0 \\ * & -\bar{\tau}_{1} Z_{1} & 0 & 0 & 0 & 0 & 0 & 0 \\ * & * & -\bar{\tau}_{2} Z_{2} & 0 & 0 & 0 & 0 & 0 \\ * & * & * & -\bar{\tau}_{2} Z_{2} & 0 & 0 & 0 & 0 \\ * & * & * & * & -\bar{P} & 0 & 0 & 0 \\ * & * & * & * & * & -\bar{P} & 0 & 0 \\ * & * & * & * & * & * & -R_{1} & 0 \\ * & * & * & * & * & * & * & -R_{2}\end{array}\right]<0$

hold for $k=1,2, \ldots, r$, where $\Omega_{k}=\left(\Omega_{i, j}^{k}\right)_{12 \times 12}$ with

$$
\begin{gathered}
\Omega_{1,1}^{k}=-P A_{k}-A_{k} P+\alpha_{0} \bar{\tau}_{1} X+\left(1-\alpha_{0}\right) \bar{\tau}_{2} X+2 Y+Q_{1}+Q_{2}+Q_{3}+Q_{4}+Q_{5}+Q_{6}, \\
\Omega_{1,2}^{k}=0, \Omega_{1,3}^{k}=-\alpha_{0} Y, \Omega_{1,4}^{k}=0, \Omega_{1,5}^{k}=-\left(1-\alpha_{0}\right) Y, \Omega_{1,6}^{k}=\Omega_{1,7}^{k}=0, \\
\Omega_{1,8}^{k}=P B_{k}+L K_{1}, \Omega_{1,9}^{k}=\alpha_{0} P W_{k}, \Omega_{1,10}^{k}=\left(1-\alpha_{0}\right) P W_{k}, \Omega_{1,11}^{k}=\Omega_{1,12}^{k}=0 \\
\Omega_{2,2}^{k}=-\left(1-\alpha_{0} \mu_{1}\right) Q_{1}, \Omega_{2,3}^{k}=0 \\
\Omega_{2,4}^{k}=\Omega_{2,5}^{k}=\Omega_{2,6}^{k}=\Omega_{2,7}^{k}=\Omega_{2,8}^{k}=\Omega_{2,9}^{k}=\Omega_{2,10}^{k}=\Omega_{2,11}^{k}=\Omega_{2,12}^{k}=0 \\
\Omega_{3,3}^{k}=-\left(1-\mu_{1}\right) Q_{5}, \Omega_{3,4}^{k}=\Omega_{3,5}^{k}=\Omega_{3,6}^{k}=\Omega_{3,7}^{k}=\Omega_{3,8}^{k}=0 \\
\Omega_{3,9}^{k}=L K_{2}, \Omega_{3,10}^{k}=\Omega_{3,11}^{k}=\Omega_{3,12}^{k}=0, \Omega_{4,4}^{k}=-Q_{2}, \\
\Omega_{4,5}^{k}=\Omega_{4,6}^{k}=\Omega_{4,7}^{k}=\Omega_{4,8}^{k}=\Omega_{4,9}^{k}=\Omega_{4,10}^{k}=\Omega_{4,11}^{k}=\Omega_{4,12}^{k}=0 \\
\Omega_{5,5}^{k}=-\left(1-\mu_{2}\right) Q_{6}, \Omega_{5,6}^{k}=0, \Omega_{5,7}^{k}=\Omega_{5,8}^{k}=\Omega_{5,9}^{k}=0, \Omega_{5,10}^{k}=L K_{3}, \\
\Omega_{5,11}^{k}=\Omega_{5,12}^{k}=0, \Omega_{6,6}^{k}=-Q_{3}, \Omega_{6,7}^{k}=\Omega_{6,8}^{k}=\Omega_{6,9}^{k}=0 \\
\Omega_{6,10}^{k}=\Omega_{6,11}^{k}=\Omega_{6,12}^{k}=0, \Omega_{7,7}^{k}=-\left(1-\alpha_{0} \mu_{2}\right) Q_{4}, \\
\Omega_{7,8}^{k}=\Omega_{7,9}^{k}=\Omega_{7,10}^{k}=\Omega_{7,11}^{k}=\Omega_{7,12}^{k}=0, \Omega_{8,8}^{k}=-2 K_{1} \\
\Omega_{8,9}^{k}=\Omega_{8,10}^{k}=\Omega_{8,11}^{k}=\Omega_{8,12}^{k}=0, \Omega_{9,9}^{k}=-2 K_{2} \\
\Omega_{9,10}^{k}=\Omega_{9,11}^{k}=\Omega_{9,12}^{k}=0, \Omega_{10,10}^{k}=-2 K_{3}, \Omega_{10,11}^{k}=\Omega_{10,12}^{k}=0
\end{gathered}
$$




$$
\begin{aligned}
& \Omega_{11,11}^{k}=-\left(\frac{1-\alpha_{0}}{\bar{\tau}_{1}}\right) Z_{1}, \Omega_{11,12}^{k}=0, \\
& \Omega_{12,12}^{k}=-\left(\frac{\alpha_{0}}{\bar{\tau}_{2}}\right) Z_{2}, \\
& \eta_{1}=\left[\begin{array}{llllllllllll}
-A_{k} & 0 & 0 & 0 & 0 & 0 & 0 & B_{k} & \alpha_{0} W_{k} & \left(1-\alpha_{0}\right) W_{k} & 0 & 0
\end{array}\right]^{T} \text {, } \\
& \eta_{2}=\left[\begin{array}{llllllllllll}
0 & 0 & 0 & 0 & 0 & 0 & 0 & 0 & W_{k} & -W_{k} & 0 & 0
\end{array}\right]^{T}, \\
& \vartheta_{1}=\left[\begin{array}{llllllllllll}
H_{0 k} & 0 & \alpha_{0} H_{1 k} & 0 & \left(1-\alpha_{0}\right) H_{1 k} & 0 & 0 & 0 & 0 & 0 & 0 & 0
\end{array}\right]^{T}, \\
& \vartheta_{2}=\left[\begin{array}{llllllllllll}
0 & 0 & H_{1 k} & 0 & -H_{1 k} & 0 & 0 & 0 & 0 & 0 & 0 & 0
\end{array}\right]^{T}, \\
& \sigma=\sqrt{\alpha_{0}\left(1-\alpha_{0}\right)}, \quad \bar{P}=P+\bar{\tau}_{1} R_{1}+\bar{\tau}_{2} R_{2} .
\end{aligned}
$$

Proof: Denoting,

$$
\begin{aligned}
y(t)= & -\bar{A} x(t)+\bar{B} f(x(t))+\alpha_{0} \bar{W} f\left(x\left(t-\tau_{1}(t)\right)\right)+\left(1-\alpha_{0}\right) \bar{W} f\left(x\left(t-\tau_{2}(t)\right)\right) \\
& +\left(\alpha(t)-\alpha_{0}\right)\left(\bar{W} f\left(x\left(t-\tau_{1}(t)\right)\right)-\bar{W} f\left(x\left(t-\tau_{2}(t)\right)\right)\right) \\
g(t)= & \bar{H}_{0} x(t)+\alpha_{0} \bar{H}_{1} x\left(t-\tau_{1}(t)\right)+\left(1-\alpha_{0}\right) \bar{H}_{1} x\left(t-\tau_{2}(t)\right) \\
& +\left(\alpha(t)-\alpha_{0}\right)\left(\bar{H}_{1} x\left(t-\tau_{1}(t)\right)-\bar{H}_{1} x\left(t-\tau_{2}(t)\right)\right) .
\end{aligned}
$$

The system (14) can be written as

$$
d x(t)=y(t) d t+g(t) d \omega(t) .
$$

Integrating (19) from $t-\tau_{1}(t)$ to $t$, and from $t-\tau_{2}(t)$ to $t$, we get the following equalities

$$
\begin{aligned}
x\left(t-\tau_{1}(t)\right) & =x(t)-\int_{t-\tau_{1}(t)}^{t} y(s) d s-\int_{t-\tau_{1}(t)}^{t} g(s) d \omega(s) \\
x\left(t-\tau_{2}(t)\right) & =x(t)-\int_{t-\tau_{2}(t)}^{t} y(s) d s-\int_{t-\tau_{2}(t)}^{t} g(s) d \omega(s),
\end{aligned}
$$

we can rewrite (14) as

$$
\begin{aligned}
d x(t)= & {\left[-\bar{A} x(t)+\bar{B} f(x(t))+\alpha_{0} \bar{W} G\left(x\left(t-\tau_{1}(t)\right)\right) x\left(t-\tau_{1}(t)\right)\right.} \\
& +\left(1-\alpha_{0}\right) \bar{W} G\left(x\left(t-\tau_{2}(t)\right)\right) x\left(t-\tau_{2}(t)\right) \\
& \left.+\left(\alpha(t)-\alpha_{0}\right)\left(\bar{W} f\left(x\left(t-\tau_{1}(t)\right)\right)-\bar{W} f\left(x\left(t-\tau_{2}(t)\right)\right)\right)\right] d t
\end{aligned}
$$




$$
\begin{aligned}
& +\left[\bar{H}_{0} x(t)+\alpha_{0} \bar{H}_{1} x\left(t-\tau_{1}(t)\right)+\left(1-\alpha_{0}\right) \bar{H}_{1} x\left(t-\tau_{2}(t)\right)\right. \\
& +\left(\alpha(t)-\alpha_{0}\right)\left(\bar{H}_{1} x\left(t-\tau_{1}(t)\right)\right. \\
& \left.\left.-\bar{H}_{1} x\left(t-\tau_{2}(t)\right)\right)\right] d \omega(t)
\end{aligned}
$$

where $G(x(t))=\operatorname{diag}\left(h_{1}\left(x_{1}(t)\right), h_{2}\left(x_{2}(t)\right), \ldots, h_{n}\left(x_{n}(t)\right)\right)$ and $0 \leq h_{j}\left(x_{j}(t)\right)=$ $f_{j}\left(x_{j}(t)\right) /\left(x_{j}(t)\right) \leq l_{j}$. Moreover, by substituting (20) and (21) into (22), we obtain

$$
\begin{gathered}
d x(t)=\left[-\bar{A} x(t)+\bar{B} f(x(t))+\alpha_{0} \bar{W} G\left(x\left(t-\tau_{1}(t)\right)\right) x(t)\right. \\
-\alpha_{0} \bar{W} G\left(x\left(t-\tau_{1}(t)\right)\right) \int_{t-\tau_{1}(t)}^{t} y(s) d s-\alpha_{0} \bar{W} G\left(x\left(t-\tau_{1}(t)\right)\right) \int_{t-\tau_{1}(t)}^{t} g(s) d \omega(s) \\
+\left(1-\alpha_{0}\right) \bar{W} G\left(x\left(t-\tau_{2}(t)\right)\right) x(t)-\left(1-\alpha_{0}\right) \bar{W} G\left(x\left(t-\tau_{2}(t)\right)\right) \int_{t-\tau_{2}(t)}^{t} y(s) d s \\
-\left(1-\alpha_{0}\right) \bar{W} G\left(x\left(t-\tau_{2}(t)\right)\right) \int_{t-\tau_{2}(t)}^{t} g(s) d \omega(s) \\
\left.+\left(\alpha(t)-\alpha_{0}\right)\left(\bar{W}_{f} f\left(x\left(t-\tau_{1}(t)\right)\right)-\bar{W} f\left(x\left(t-\tau_{2}(t)\right)\right)\right)\right] d t \\
+\left[\bar{H}_{0} x(t)+\alpha_{0} \bar{H}_{1} x\left(t-\tau_{1}(t)\right)+\left(1-\alpha_{0}\right) \bar{H}_{1} x\left(t-\tau_{2}(t)\right)+\right. \\
\left.\left(\alpha(t)-\alpha_{0}\right)\left(\bar{H}_{1} x\left(t-\tau_{1}(t)\right)-\bar{H}_{1} x\left(t-\tau_{2}(t)\right)\right)\right] d \omega(t) .
\end{gathered}
$$

Choose a Lyapunov-Krasovskii functional candidate as follows

$$
\begin{gathered}
V(x(t), t)=x^{T}(t) P x(t)+\int_{t-\alpha_{0} \tau_{1}(t)}^{t} x^{T}(s) Q_{1} x(s) d s+\int_{t-\tau_{0}}^{t} x^{T}(s) Q_{2} x(s) d s \\
+\int_{t-\bar{\tau}}^{t} x^{T}(s) Q_{3} x(s) d s+\int_{t-\alpha_{0} \tau_{2}(t)}^{t} x^{T}(s) Q_{4} x(s) d s+\int_{t-\tau_{1}(t)}^{t} x^{T}(s) Q_{5} x(s) d s \\
+\int_{t-\tau_{2}(t)}^{t} x^{T}(s) Q_{6} x(s) d s+\int_{-\bar{\tau}_{1}}^{0} \int_{t+\beta}^{t} y^{T}(\alpha) Z_{1} y(\alpha) d \alpha d \beta+\int_{-\bar{\tau}_{2}}^{0} \int_{t+\beta}^{t} y^{T}(\alpha) Z_{2} y(\alpha) d \alpha d \beta
\end{gathered}
$$




$$
+\int_{-\bar{\tau}_{1}}^{0} \int_{t+\beta}^{t} g^{T}(\alpha) R_{1} g(\alpha) d \alpha d \beta+\int_{-\bar{\tau}_{2}}^{0} \int_{t+\beta}^{t} g^{T}(\alpha) R_{2} g(\alpha) d \alpha d \beta .
$$

By Ito's formula, we can calculate $\mathcal{L} V(x(t), t)$ along with $(24)$, then we have

$$
d V(x(t), t)=\mathcal{L} V(x(t), t)+2 x^{T}(t) P g(t) d \omega(t),
$$

where

$$
\begin{aligned}
& \mathcal{L} V(x(t), t) \leq 2 x^{T}(t) P\left[-\bar{A} x(t)+\bar{B} f(x(t))+\alpha_{0} \bar{W} G\left(x\left(t-\tau_{1}(t)\right)\right) x(t)\right. \\
& -\alpha_{0} \bar{W} G\left(x\left(t-\tau_{1}(t)\right)\right) \int_{t-\tau_{1}(t)}^{t} y(s) d s-\alpha_{0} \bar{W} G\left(x\left(t-\tau_{1}(t)\right)\right) \int_{t-\tau_{1}(t)}^{t} g(s) d \omega(s) \\
& +\left(1-\alpha_{0}\right) \bar{W} G\left(x\left(t-\tau_{2}(t)\right)\right) x(t)-\left(1-\alpha_{0}\right) \bar{W} G\left(x\left(t-\tau_{2}(t)\right)\right) \int_{t-\tau_{2}(t)}^{t} y(s) d s \\
& -\left(1-\alpha_{0}\right) \bar{W} G\left(x\left(t-\tau_{2}(t)\right)\right) \int_{t-\tau_{2}(t)}^{t} g(s) d \omega(s) \\
& \left.+\left(\alpha(t)-\alpha_{0}\right)\left(\bar{W} f\left(x\left(t-\tau_{1}(t)\right)\right)-\bar{W} f\left(x\left(t-\tau_{2}(t)\right)\right)\right)\right] \\
& +g^{T}(t) P g(t)+x^{T}(t) Q_{1} x(t)-\left(1-\alpha_{0} \mu_{1}\right) x^{T}\left(t-\alpha_{0} \tau_{1}(t)\right) Q_{1} x\left(t-\alpha_{0} \tau_{1}(t)\right) \\
& +x^{T}(t) Q_{2} x(t)-x^{T}\left(t-\tau_{0}\right) Q_{2} x\left(t-\tau_{0}\right)+x^{T}(t) Q_{3} x(t)-x^{T}(t-\bar{\tau}) Q_{3} x(t-\bar{\tau}) \\
& +x^{T}(t) Q_{4} x(t)-\left(1-\alpha_{0} \mu_{2}\right) x^{T}\left(t-\alpha_{0} \tau_{2}(t)\right) Q_{4} x\left(t-\alpha_{0} \tau_{2}(t)\right) \\
& +x^{T}(t) Q_{5} x(t)-\left(1-\mu_{1}\right) x^{T}\left(t-\tau_{1}(t)\right) Q_{5} x\left(t-\tau_{1}(t)\right)+x^{T}(t) Q_{6} x(t) \\
& -\left(1-\mu_{2}\right) x^{T}\left(t-\tau_{2}(t)\right) Q_{6} x\left(t-\tau_{2}(t)\right) \\
& +\bar{\tau}_{1} y^{T}(t) Z_{1} y(t)-\int_{t-\bar{\tau}_{1}}^{t} y^{T}(s) Z_{1} y(s) d s \\
& +\bar{\tau}_{2} y^{T}(t) Z_{2} y(t)-\int_{t-\bar{\tau}_{2}}^{t} y^{T}(s) Z_{2} y(s) d s+\bar{\tau}_{1} g^{T}(t) R_{1} g(t)
\end{aligned}
$$




$$
-\int_{t-\bar{\tau}_{1}}^{t} g^{T}(s) R_{1} g(s) d s+\bar{\tau}_{2} g^{T}(t) R_{2} g(t)-\int_{t-\bar{\tau}_{2}}^{t} g^{T}(s) R_{2} g(s) d s .
$$

Define $a(\cdot), b(\cdot)$ and $N$ in Lemma 2.11 as $a(\alpha)=x(t), b(\alpha)=y(s), N=P \bar{W} G(x(t-$ $\left.\left.\tau_{1}(t)\right)\right)$ and using $(20)$, then

$$
\begin{aligned}
& -2 \alpha_{0} x^{T}(t) P \bar{W} G\left(x\left(t-\tau_{1}(t)\right)\right) \int_{t-\tau_{1}(t)}^{t} y(s) d s \\
& \leq \alpha_{0} \int_{t-\tau_{1}(t)}^{t}\left[\begin{array}{c}
x(t) \\
y(s)
\end{array}\right]^{T}\left[\begin{array}{cc}
X & Y-P \bar{W} G\left(x\left(t-\tau_{1}(t)\right)\right) \\
Z_{1}
\end{array}\right]\left[\begin{array}{c}
x(t) \\
y(s)
\end{array}\right] d s \\
& \leq \alpha_{0}\left\{\bar{\tau}_{1} x^{T}(t) X x(t)+2 x^{T}(t)\left[Y-P \bar{W} G\left(x\left(t-\tau_{1}(t)\right)\right)\right] \int_{t-\tau_{1}(t)}^{t} y(s) d s\right. \\
& \left.\quad+\int_{t-\tau_{1}(t)}^{t} y^{T}(s) Z_{1} y(s) d s\right\} \\
& \leq \alpha_{0} \bar{\tau}_{1} x^{T}(t) X x(t)+2 \alpha_{0} x^{T}(t) Y x(t)-2 \alpha_{0} x^{T}(t) Y x\left(t-\tau_{1}(t)\right) \\
& -2 \alpha_{0} x^{T}(t) Y \int_{t-\tau_{1}(t)}^{t} g(s) d \omega(s)-2 \alpha_{0} x^{T}(t) P \bar{W} G\left(x\left(t-\tau_{1}(t)\right)\right) x(t) \\
& 2+\alpha_{0} x^{T}(t) P \bar{W} f\left(x\left(t-\tau_{1}(t)\right)\right)+2 \alpha_{0} x^{T}(t) P \bar{W} G\left(x\left(t-\tau_{1}(t)\right)\right) \int_{t-\tau_{1}(t)}^{t} g(s) d \omega(s) \\
& \int_{t-\tau_{1}(t)}^{t} y^{T}(s) Z_{1} y(s) d s .
\end{aligned}
$$

Define $a(),. b($.$) and N$ in Lemma 2.11 as $a(\alpha)=x(t), b(\alpha)=y(s), N=P \bar{W} G(x(t-$ $\left.\left.\tau_{2}(t)\right)\right)$ and using $(21)$, then

$$
-2\left(1-\alpha_{0}\right) x^{T}(t) P \bar{W} G\left(x\left(t-\tau_{2}(t)\right)\right) \int_{t-\tau_{2}(t)}^{t} y(s) d s
$$




$$
\begin{gathered}
\leq \quad\left(1-\alpha_{0}\right) \int_{t-\tau_{2}(t)}^{t}\left[\begin{array}{c}
x(t) \\
y(s)
\end{array}\right]^{T}\left[\begin{array}{cc}
X & Y-P \bar{W} G\left(x\left(t-\tau_{2}(t)\right)\right) \\
Z_{2}
\end{array}\right]\left[\begin{array}{l}
x(t) \\
y(s)
\end{array}\right] d s \\
\leq \quad\left(1-\alpha_{0}\right) \bar{\tau}_{2} x^{T}(t) X x(t)+2\left(1-\alpha_{0}\right) x^{T}(t) Y x(t)-2\left(1-\alpha_{0}\right) x^{T}(t) Y x\left(t-\tau_{2}(t)\right) \\
-2\left(1-\alpha_{0}\right) x^{T}(t) Y \int_{t-\tau_{2}(t)}^{t} g(s) d \omega(s)-2\left(1-\alpha_{0}\right) x^{T}(t) P \bar{W} G\left(x\left(t-\tau_{2}(t)\right)\right) x(t) \\
+2\left(1-\alpha_{0}\right) x^{T}(t) P \bar{W} f\left(x\left(t-\tau_{2}(t)\right)\right)+2\left(1-\alpha_{0}\right) x^{T}(t) P \bar{W} G\left(x\left(t-\tau_{2}(t)\right)\right) \\
\times \int_{t-\tau_{2}(t)}^{t} g(s) d \omega(s)+\left(1-\alpha_{0}\right) \int_{t-\tau_{2}(t)}^{t} y^{T}(s) Z_{2} y(s) d s
\end{gathered}
$$

Here

$$
\begin{aligned}
& f\left(x\left(t-\tau_{1}(t)\right)\right)=G\left(x\left(t-\tau_{1}(t)\right)\right) x\left(t-\tau_{1}(t)\right), \quad \text { and } \\
& f\left(x\left(t-\tau_{2}(t)\right)\right)=G\left(x\left(t-\tau_{2}(t)\right)\right) x\left(t-\tau_{2}(t)\right)
\end{aligned}
$$

are used. Using (27) and (28) in (26), we have

$$
\begin{gathered}
\mathcal{L} V(x(t), t) \leq-2 x^{T}(t) P \bar{A} x(t)+2 x^{T}(t) P \bar{B} f(x(t))+\alpha_{0} \bar{\tau}_{1} x^{T}(t) X x(t) \\
-2 \alpha_{0} x^{T}(t) Y x\left(t-\tau_{1}(t)\right)-2 \alpha_{0} x^{T}(t) Y \int_{t-\tau_{1}(t)}^{t} g(s) d \omega(s) \\
+2 \alpha_{0} x^{T}(t) P \bar{W} f\left(x\left(t-\tau_{1}(t)\right)\right)-\left(1-\alpha_{0}\right) \int_{t-\tau_{1}(t)}^{t} y^{T}(s) Z_{1} y(s) d s \\
+\left(1-\alpha_{0}\right) \bar{\tau}_{2} x^{T}(t) X x(t)+2 x^{T}(t) Y x(t)-2\left(1-\alpha_{0}\right) x^{T}(t) Y x\left(t-\tau_{2}(t)\right) \\
-2\left(1-\alpha_{0}\right) x^{T}(t) Y \int_{t-\tau_{2}(t)}^{t} g(s) d \omega(s)+2\left(1-\alpha_{0}\right) x^{T}(t) P \bar{W} f\left(x\left(t-\tau_{2}(t)\right)\right) \\
-\alpha_{0} \int_{t-\tau_{2}(t)}^{t} y^{T}(s) Z_{2} y(s) d s+2\left(\alpha(t)-\alpha_{0}\right) \\
\times x^{T}(t) P\left(\bar{W} f\left(x\left(t-\tau_{1}(t)\right)\right)-\bar{W} f\left(x\left(t-\tau_{2}(t)\right)\right)\right)
\end{gathered}
$$




$$
\begin{gathered}
+g^{T}(t)\left(P+\bar{\tau}_{1} R_{1}+\bar{\tau}_{2} R_{2}\right) g(t)+x^{T}(t) Q_{1} x(t) \\
-\left(1-\alpha_{0} \mu_{1}\right) x^{T}\left(t-\alpha_{0} \tau_{1}(t)\right) Q_{1} x\left(t-\alpha_{0} \tau_{1}(t)\right) \\
+x^{T}(t) Q_{2} x(t)-x^{T}\left(t-\tau_{0}\right) Q_{2} x\left(t-\tau_{0}\right)+x^{T}(t) Q_{3} x(t)-x^{T}(t-\bar{\tau}) Q_{3} x(t-\bar{\tau}) \\
+x^{T}(t) Q_{4} x(t)-\left(1-\alpha_{0} \mu_{2}\right) x^{T}\left(t-\alpha_{0} \tau_{2}(t)\right) Q_{4} x\left(t-\alpha_{0} \tau_{2}(t)\right)+x^{T}(t) Q_{5} x(t) \\
-\left(1-\mu_{1}\right) x^{T}\left(t-\tau_{1}(t)\right) Q_{5} x\left(t-\tau_{1}(t)\right) \\
+x^{T}(t) Q_{6} x(t)-\left(1-\mu_{2}\right) x^{T}\left(t-\tau_{2}(t)\right) Q_{6} x\left(t-\tau_{2}(t)\right) \\
+y^{T}(t)\left(\bar{\tau}_{1} Z_{1}+\bar{\tau}_{2} Z_{2}\right) y(t) \\
-\int_{t-\tau_{1}(t)}^{t} g^{T}(s) R_{1} g(s) d s-\int_{t-\tau_{2}(t)}^{t} g^{T}(s) R_{2} g(s) d s .
\end{gathered}
$$

For symmetric positive definite matrices $R_{1}$, and $R_{2}$, it follows from Lemma 2.7 that

$$
\begin{gathered}
\frac{1}{\alpha_{0}}\left\{-2 x^{T}(t) Y \int_{t-\tau_{1}(t)}^{t} g(s) d \omega(s)\right\} \leq \frac{1}{\alpha_{0}}\left\{x^{T}(t) Y R_{1}^{-1} Y^{T} x(t)\right. \\
\left.+\left(\int_{t-\tau_{1}(t)}^{t} g(s) d \omega(s)\right)^{T} R_{1}\left(\int_{t-\tau_{1}(t)}^{t} g(s) d \omega(s)\right)\right\}, \\
\frac{1}{1-\alpha_{0}}\left\{-2 x^{T}(t) Y \int_{t-\tau_{2}(t)}^{t} g(s) d \omega(s)\right\} \leq \frac{1}{1-\alpha_{0}}\left\{x^{T}(t) Y R_{2}^{-1} Y^{T} x(t)\right. \\
\left.+\left(\int_{t-\tau_{2}(t)}^{t} g(s) d \omega(s)\right)^{T} R_{2}\left(\int_{t-\tau_{2}(t)}^{t} g(s) d \omega(s)\right)\right\} .
\end{gathered}
$$

It is clear from (3) that

$$
\begin{aligned}
f_{j}\left(x_{j}(t)\right)\left[f_{j}\left(x_{j}(t)\right)-l_{j} x_{j}(t)\right] & \leq 0 \\
f_{j}\left(x_{j}\left(t-\tau_{i}(t)\right)\right)\left[f_{j}\left(x_{j}\left(t-\tau_{i}(t)\right)\right)-l_{j} x_{j}\left(t-\tau_{i}(t)\right)\right] & \leq 0, \quad j=1,2, \ldots, n .
\end{aligned}
$$


From inequalities (32) and (33), for any positive diagonal matrices $K_{i}=\operatorname{diag}\left(k_{i 1}, k_{i 2}, \ldots, k_{i n}\right)$, $i=1,2,3$, the following inequalities hold

$$
\begin{aligned}
0 \leq & -2 \sum_{j=1}^{n} k_{1 j} f_{j}\left(x_{j}(t)\right)\left[f_{j}\left(x_{j}(t)\right)-l_{j} x_{j}(t)\right]-2 \sum_{i=1}^{2} \sum_{j=1}^{n} k_{(i+1) j} f_{j}\left(x_{j}\left(t-\tau_{i}(t)\right)\right) \\
& \times\left[f_{j}\left(x_{j}\left(t-\tau_{i}(t)\right)\right)-l_{j} x_{j}\left(t-\tau_{i}(t)\right)\right] \\
= & 2 x^{T}(t) L K_{1} f(x(t))-2 f^{T}(x(t)) K_{1} f(x(t)) \\
& +2 x^{T}\left(t-\tau_{1}(t)\right) L K_{2} f\left(x\left(t-\tau_{1}(t)\right)\right)-2 f^{T}\left(x\left(t-\tau_{1}(t)\right)\right) \\
& K_{2} f\left(x\left(t-\tau_{1}(t)\right)\right)+2 x^{T}\left(t-\tau_{2}(t)\right) L K_{3} f\left(x\left(t-\tau_{2}(t)\right)\right) \\
& -2 f^{T}\left(x\left(t-\tau_{2}(t)\right)\right) K_{3} f\left(x\left(t-\tau_{2}(t)\right)\right) .
\end{aligned}
$$

where $L=\operatorname{diag}\left(l_{1}, l_{2}, \ldots, l_{n}\right)$. By Remark 2.4 , it is easy to know

$$
\begin{gathered}
\mathbb{E}\left\{2\left(\alpha(t)-\alpha_{0}\right) x^{T}(t) P\left(\bar{W} f\left(x\left(t-\tau_{1}(t)\right)\right)-\bar{W} f\left(x\left(t-\tau_{2}(t)\right)\right)\right)\right\}=0 \\
\mathbb{E}\left\{y^{T}(t) Z_{1} y(t)\right\}=\mathbb{E}\left\{\left[-\bar{A} x(t)+\bar{B} f(x(t))+\alpha_{0} \bar{W} f\left(x\left(t-\tau_{1}(t)\right)\right)\right.\right. \\
\left.+\left(1-\alpha_{0}\right) \bar{W} f\left(x\left(t-\tau_{2}(t)\right)\right)\right]^{T} \\
\times Z_{1}\left[-\bar{A} x(t)+\bar{B} f(x(t))+\alpha_{0} \bar{W} f\left(x\left(t-\tau_{1}(t)\right)\right)+\left(1-\alpha_{0}\right) \bar{W} f\left(x\left(t-\tau_{2}(t)\right)\right)\right] \\
+2\left(\alpha(t)-\alpha_{0}\right)\left[-\bar{A} x(t)+\bar{B} f(x(t))+\alpha_{0} \bar{W} f\left(x\left(t-\tau_{1}(t)\right)\right)+\left(1-\alpha_{0}\right) \bar{W}\right. \\
\left.\times f\left(x\left(t-\tau_{2}(t)\right)\right)\right] Z_{1}\left[\bar{W} f\left(x\left(t-\tau_{1}(t)\right)\right)-\bar{W} f\left(x\left(t-\tau_{2}(t)\right)\right)\right]+\left(\alpha(t)-\alpha_{0}\right)^{2} \\
\times\left[\bar{W} f\left(x\left(t-\tau_{1}(t)\right)\right)-\bar{W} f\left(x\left(t-\tau_{2}(t)\right)\right)\right]^{T} Z_{1} \\
\left.\times\left[\bar{W} f\left(x\left(t-\tau_{1}(t)\right)\right)-\bar{W} f\left(x\left(t-\tau_{2}(t)\right)\right)\right]\right\} \\
=\left[-\bar{A} x(t)+\bar{B} f(x(t))+\alpha_{0} \bar{W} f\left(x\left(t-\tau_{1}(t)\right)\right)+\left(1-\alpha_{0}\right) \bar{W} f\left(x\left(t-\tau_{2}(t)\right)\right)\right]^{T} Z_{1} \\
\times\left[-\bar{A} x(t)+\bar{B} f(x(t))+\alpha_{0} \bar{W} f\left(x\left(t-\tau_{1}(t)\right)\right)+\left(1-\alpha_{0}\right) \bar{W} f\left(x\left(t-\tau_{2}(t)\right)\right)\right] \\
+\alpha_{0}\left(1-\alpha_{0}\right)\left[\bar{W} f\left(x\left(t-\tau_{1}(t)\right)\right)-\bar{W} f\left(x\left(t-\tau_{2}(t)\right)\right)\right]^{T} Z_{1} \\
\times\left[\bar{W} f\left(x\left(t-\tau_{1}(t)\right)\right)-\bar{W} f\left(x\left(t-\tau_{2}(t)\right)\right)\right]
\end{gathered}
$$




$$
\mathbb{E}\left\{y^{T}(t) Z_{1} y(t)\right\}=\zeta^{T}(t)\left\{\eta_{1} Z_{1} \eta_{1}^{T}+\alpha_{0}\left(1-\alpha_{0}\right) \eta_{2} Z_{1} \eta_{2}^{T}\right\} \zeta(t) .
$$

Similarly we have,

$$
\mathbb{E}\left\{y^{T}(t) Z_{2} y(t)\right\}=\zeta^{T}(t)\left\{\eta_{1} Z_{2} \eta_{1}^{T}+\alpha_{0}\left(1-\alpha_{0}\right) \eta_{2} Z_{2} \eta_{2}^{T}\right\} \zeta(t),
$$

and

$$
\begin{aligned}
\mathbb{E}\left\{g^{T}(t)\left(P+\bar{\tau}_{1} R_{1}+\bar{\tau}_{2} R_{2}\right) g(t)\right\}=\zeta^{T}(t)\left\{\vartheta_{1} \bar{P} \vartheta_{1}^{T}+\alpha_{0}\left(1-\alpha_{0}\right) \vartheta_{2} \bar{P} \vartheta_{2}^{T}\right\} \zeta(t) . \\
\mathbb{E}\left\{\left(\int_{t-\tau_{1}(t)}^{t} g(s) d \omega(s)\right)^{T} R_{1}\left(\int_{t-\tau_{1}(t)}^{t} g(s) d \omega(s)\right)\right\}=\mathbb{E}\left\{\int_{t-\tau_{1}(t)}^{t} g^{T}(s) R_{1} g(s) d s\right\} \\
\mathbb{E}\left\{\left(\int_{t-\tau_{2}(t)}^{t} g(s) d \omega(s)\right)^{T} R_{2}\left(\int_{t-\tau_{2}(t)}^{t} g(s) d \omega(s)\right)\right\}=\mathbb{E}\left\{\int_{t-\tau_{2}(t)}^{t} g^{T}(s) R_{2} g(s) d s\right\} .
\end{aligned}
$$

Using Lemma 2.8 in (29) and substituting (29)-(31), (34) into (25) and taking mathematical expectation on both sides of (25) then using (35)-(40), we can get

$$
\begin{gathered}
\mathbb{E} d V(x(t), t)=\mathbb{E}\{\mathcal{L} V(x(t), t)\} \\
\leq \zeta^{T}(t)\left\{\bar{\Omega}+\bar{\tau}_{1} \eta_{1} Z_{1} \eta_{1}^{T}+\bar{\tau}_{1} \alpha_{0}\left(1-\alpha_{0}\right) \eta_{2} Z_{1} \eta_{2}^{T}+\bar{\tau}_{2} \eta_{1} Z_{2} \eta_{1}^{T}\right. \\
+\bar{\tau}_{2} \alpha_{0}\left(1-\alpha_{0}\right) \eta_{2} Z_{2} \eta_{2}^{T}+\vartheta_{1} \bar{P} \vartheta_{1}^{T}+\alpha_{0}\left(1-\alpha_{0}\right) \vartheta_{2} \bar{P} \vartheta_{2}^{T} \\
\left.+Y R_{1}^{-1} Y^{T}+Y R_{2}^{-1} Y^{T}\right\} \zeta(t),
\end{gathered}
$$

where

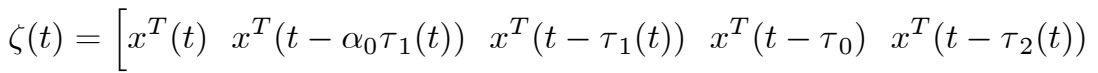

$$
\begin{aligned}
& x^{T}(t-\bar{\tau}) x^{T}\left(t-\alpha_{0} \tau_{2}(t)\right) f^{T}(x(t)) f^{T}\left(x\left(t-\tau_{1}(t)\right)\right) \quad f^{T}\left(x\left(t-\tau_{2}(t)\right)\right) \\
& \left.\left(\int_{t-\tau_{1}(t)}^{t} y(s) d s\right)^{T}\left(\int_{t-\tau_{2}(t)}^{t} y(s) d s\right)^{T}\right]^{T} .
\end{aligned}
$$

Let us define,

$$
\begin{gathered}
\bar{\Upsilon}=\bar{\Omega}+\bar{\tau}_{1} \eta_{1} Z_{1} \eta_{1}^{T}+\bar{\tau}_{1} \alpha_{0}\left(1-\alpha_{0}\right) \eta_{2} Z_{1} \eta_{2}^{T}+\bar{\tau}_{2} \eta_{1} Z_{2} \eta_{1}^{T} \\
+\bar{\tau}_{2} \alpha_{0}\left(1-\alpha_{0}\right) \eta_{2} Z_{2} \eta_{2}^{T}+\vartheta_{1} \bar{P} \vartheta_{1}^{T}+\alpha_{0}\left(1-\alpha_{0}\right) \vartheta_{2} \bar{P} \vartheta_{2}^{T} \\
+Y R_{1}^{-1} Y^{T}+Y R_{2}^{-1} Y^{T}<0 .
\end{gathered}
$$


Considering $\mu_{k}(\theta(t)) \geq 0(k=1,2, \ldots, r)$ and $\Upsilon_{k}<0(k=1,2, \ldots, r)$ in Theorem 3.1 , we have $\sum_{k=1}^{r} \mu_{k}(\theta(t)) \Upsilon_{k}<0$. Noting that $\sum_{k=1}^{r} \mu_{k}(\theta(t))=1$. Where

$$
\begin{aligned}
\Upsilon_{k}=\Omega_{k}+ & \bar{\tau}_{1} \eta_{1} Z_{1} \eta_{1}^{T}+\bar{\tau}_{1} \alpha_{0}\left(1-\alpha_{0}\right) \eta_{2} Z_{1} \eta_{2}^{T}+\bar{\tau}_{2} \eta_{1} Z_{2} \eta_{1}^{T} \\
& +\bar{\tau}_{2} \alpha_{0}\left(1-\alpha_{0}\right) \eta_{2} Z_{2} \eta_{2}^{T}+\vartheta_{1} \bar{P} \vartheta_{1}^{T} \\
& +\alpha_{0}\left(1-\alpha_{0}\right) \vartheta_{2} \bar{P} \vartheta_{2}^{T}+Y R_{1}^{-1} Y^{T}+Y R_{2}^{-1} Y^{T}<0,
\end{aligned}
$$

$\Omega_{k}, \eta_{1}, \eta_{2}, \vartheta_{1}$, and $\vartheta_{2}$ are defined as in Theorem 3.1. By Schur complement, we know that $\Upsilon_{k}<0$ is equivalent to (16). Let $\lambda=\min \left\{\lambda_{\min }\left(-\Upsilon_{k}\right)\right\}$, then by the generalized Ito's formula [13], we have

$$
\mathbb{E} V(x(t), t)-\mathbb{E} V(x(0), 0)=\mathbb{E} \int_{0}^{t} \mathcal{L} V(x(s), s) d s \leq-\lambda \mathbb{E} \int_{0}^{t}\|x(s)\|^{2} d s .
$$

Moreover,

$$
\mathbb{E} \int_{0}^{t}\|x(s)\|^{2} d s \leq \frac{1}{\lambda} \mathbb{E} V(x(0), 0), \quad t \geq 0 .
$$

which indicates that system (14) is globally asymptotically stable in the mean square. This completes the proof.

In the following part, we extend the above result to uncertain fuzzy stochastic Hopfield neural network (UFSHNN) (12) and obtain the stability criteria as the following theorem by means of the feasibility of LMIs.

Theorem 3.2 For given scalars $\bar{\tau}_{1}, \bar{\tau}_{2}, \mu_{1}, \mu_{2}$, and $0<\alpha_{0}<1$ satisfying $\alpha_{0} \mu_{1}<1$, the UFSHNN (12) is globally robustly asymptotically stable in the mean square, if there exist symmetric positive definite matrices $P>0, Q_{i}>0$ $(i=1,2,3,4,5,6), R_{1}>0, R_{2}>0, Z_{1}>0$, and $Z_{2}>0$, for any matrices $X$ and $Y$, positive diagonal matrices $K_{1}>0, K_{2}>0, K_{3}>0$ and positive scalars $\epsilon_{j}>0$ $(j=1, \ldots, 7)$, such that the following LMIs

$$
\begin{aligned}
& {\left[\begin{array}{ll}
X & Y \\
& Z_{1}
\end{array}\right] \geq 0 \text { and }\left[\begin{array}{ll}
X & Y \\
& Z_{2}
\end{array}\right] \geq 0} \\
& {\left[\begin{array}{cccccccc}
\Upsilon_{k} & \Gamma_{1} & \bar{\tau}_{1} \Gamma_{2} & \bar{\tau}_{1} \Gamma_{3} & \bar{\tau}_{2} \Gamma_{4} & \bar{\tau}_{2} \Gamma_{5} & \Gamma_{6} & \Gamma_{7} \\
& -\epsilon_{1} I & 0 & 0 & 0 & 0 & 0 & 0 \\
* & -\bar{\tau}_{1} \epsilon_{2} & 0 & 0 & 0 & 0 & 0 \\
& * & * & -\bar{\tau}_{1} \epsilon_{3} & 0 & 0 & 0 & 0 \\
* & * & * & -\bar{\tau}_{2} \epsilon_{4} & 0 & 0 & 0 \\
& * & * & * & -\bar{\tau}_{2} \epsilon_{5} & 0 & 0 \\
* & * & * & * & * & -\epsilon_{6} I & 0 \\
& * & * & * & * & * & * & -\epsilon_{7} I \\
& & & & & & &
\end{array}\right]<0}
\end{aligned}
$$


hold for $k=1,2, \ldots, r$, where $\Upsilon_{k}$ and $\sigma$ are defined as in Theorem 3.1, with

$$
\begin{aligned}
& \Omega_{1,1}^{k}=-P A_{k}-A_{k} P+\alpha_{0} \bar{\tau}_{1} X+\left(1-\alpha_{0}\right) \bar{\tau}_{2} X+2 Y+Q_{1}+Q_{2}+Q_{3}+Q_{4}+Q_{5}+Q_{6} \\
& +\left(\epsilon_{1}+\bar{\tau}_{1} \epsilon_{2}+\bar{\tau}_{2} \epsilon_{4}\right)\left(E_{k}^{A}\right)^{T}\left(E_{k}^{A}\right)+\epsilon_{6}\left(E_{k}^{H_{0}}\right)^{T}\left(E_{k}^{H_{0}}\right), \Omega_{1,2}^{k}=0, \\
& \Omega_{1,3}^{k}=-\alpha_{0} Y+\alpha_{0} \epsilon_{6}\left(E_{k}^{H_{0}}\right)^{T}\left(E_{k}^{H_{1}}\right), \Omega_{1,4}^{k}=0, \\
& \Omega_{1,5}^{k}=-\left(1-\alpha_{0}\right) Y+\left(1-\alpha_{0}\right) \epsilon_{6}\left(E_{k}^{H_{0}}\right)^{T}\left(E_{k}^{H_{1}}\right), \Omega_{1,6}^{k}=0, \\
& \Omega_{1,7}^{k}=0, \Omega_{1,8}^{k}=P B_{k}+L K_{1}-\left(\epsilon_{1}+\bar{\tau}_{1} \epsilon_{2}+\bar{\tau}_{2} \epsilon_{4}\right)\left(E_{k}^{A}\right)^{T}\left(E_{k}^{B}\right), \\
& \Omega_{1,9}^{k}=\alpha_{0} P W_{k}-\alpha_{0}\left(\epsilon_{1}+\bar{\tau}_{1} \epsilon_{2}+\bar{\tau}_{2} \epsilon_{4}\right)\left(E_{k}^{A}\right)^{T}\left(E_{k}^{W}\right), \\
& \Omega_{1,10}^{k}=\left(1-\alpha_{0}\right) P W_{k}-\left(1-\alpha_{0}\right)\left(\epsilon_{1}+\bar{\tau}_{1} \epsilon_{2}+\bar{\tau}_{2} \epsilon_{4}\right)\left(E_{k}^{A}\right)^{T}\left(E_{k}^{W}\right), \Omega_{1,11}^{k}=0, \\
& \Omega_{1,12}^{k}=0, \Omega_{2,2}^{k}=-\left(1-\alpha_{0} \mu_{1}\right) Q_{1}, \\
& \Omega_{2,3}^{k}=\Omega_{2,4}^{k}=\Omega_{2,5}^{k}=\Omega_{2,6}^{k}=\Omega_{2,7}^{k}=\Omega_{2,8}^{k}=\Omega_{2,9}^{k}=\Omega_{2,10}^{k}=0, \\
& \Omega_{2,11}^{k}=\Omega_{2,12}^{k}=0, \Omega_{3,3}^{k}=-\left(1-\mu_{1}\right) Q_{5}+\alpha_{0}^{2} \epsilon_{6}\left(E_{k}^{H_{1}}\right)^{T}\left(E_{k}^{H_{1}}\right)+\sigma^{2} \epsilon_{7}\left(E_{k}^{H_{1}}\right)^{T}\left(E_{k}^{H_{1}}\right) \text {, } \\
& \Omega_{3,4}^{k}=0, \Omega_{3,5}^{k}=\sigma^{2} \epsilon_{6}\left(E_{k}^{H_{1}}\right)^{T}\left(E_{k}^{H_{1}}\right)+\sigma^{2} \epsilon_{7}\left(E_{k}^{H_{1}}\right)^{T}\left(E_{k}^{H_{1}}\right), \\
& \Omega_{3,6}^{k}=\Omega_{3,7}^{k}=\Omega_{3,8}^{k}=0, \Omega_{3,9}^{k}=L K_{2}, \Omega_{3,10}^{k}=0, \Omega_{3,11}^{k}=\Omega_{3,12}^{k}=0, \\
& \Omega_{4,4}^{k}=-Q_{2}, \Omega_{4,5}^{k}=\Omega_{4,6}^{k}=\Omega_{4,7}^{k}=\Omega_{4,8}^{k}=\Omega_{4,9}^{k}=\Omega_{4,10}^{k}=\Omega_{4,11}^{k}=\Omega_{4,12}^{k}=0, \\
& \Omega_{5,5}^{k}=-\left(1-\mu_{2}\right) Q_{6}+\left(1-\alpha_{0}\right)^{2} \epsilon_{6}\left(E_{k}^{H_{1}}\right)^{T}\left(E_{k}^{H_{1}}\right)+\sigma^{2} \epsilon_{7}\left(E_{k}^{H_{1}}\right)^{T}\left(E_{k}^{H_{1}}\right), \\
& \Omega_{5,6}^{k}=\Omega_{5,7}^{k}=\Omega_{5,8}^{k}=\Omega_{5,9}^{k}=0, \Omega_{5,10}^{k}=L K_{3}, \Omega_{5,11}^{k}=\Omega_{5,12}^{k}=0, \\
& \Omega_{6,6}^{k}=-Q_{3}, \Omega_{6,7}^{k}=\Omega_{6,8}^{k}=\Omega_{6,9}^{k}=\Omega_{6,10}^{k}=\Omega_{6,11}^{k}=\Omega_{6,12}^{k}=0, \\
& \Omega_{7,7}^{k}=-\left(1-\alpha_{0} \mu_{2}\right) Q_{4}, \Omega_{7,8}^{k}=\Omega_{7,9}^{k}=\Omega_{7,10}^{k}=\Omega_{7,11}^{k}=\Omega_{7,12}^{k}=0 \text {, } \\
& \Omega_{8,8}^{k}=-2 K_{1}+\left(\epsilon_{1}+\bar{\tau}_{1} \epsilon_{2}+\bar{\tau}_{2} \epsilon_{4}\right)\left(E_{k}^{B}\right)^{T}\left(E_{k}^{B}\right), \\
& \Omega_{8,9}^{k}=\alpha_{0}\left(\epsilon_{1}+\bar{\tau}_{1} \epsilon_{2}+\bar{\tau}_{2} \epsilon_{4}\right)\left(E_{k}^{B}\right)^{T}\left(E_{k}^{W}\right), \\
& \Omega_{8,10}^{k}=\left(1-\alpha_{0}\right)\left(\epsilon_{1}+\bar{\tau}_{1} \epsilon_{2}+\bar{\tau}_{2} \epsilon_{4}\right)\left(E_{k}^{B}\right)^{T}\left(E_{k}^{W}\right), \Omega_{8,11}^{k}=\Omega_{8,12}^{k}=0, \\
& \Omega_{9,9}^{k}=-2 K_{2}+\alpha_{0}^{2}\left(\epsilon_{1}+\bar{\tau}_{1} \epsilon_{2}+\bar{\tau}_{2} \epsilon_{4}\right)\left(E_{k}^{W}\right)^{T}\left(E_{k}^{W}\right)+\sigma^{2}\left(\bar{\tau}_{1} \epsilon_{3}+\bar{\tau}_{2} \epsilon_{5}\right)\left(E_{k}^{W}\right)^{T}\left(E_{k}^{W}\right) \text {, } \\
& \Omega_{9,10}^{k}=\sigma^{2}\left(\epsilon_{1}+\bar{\tau}_{1} \epsilon_{2}+\bar{\tau}_{2} \epsilon_{4}\right)\left(E_{k}^{W}\right)^{T}\left(E_{k}^{W}\right)-\sigma^{2}\left(\bar{\tau}_{1} \epsilon_{3}+\bar{\tau}_{2} \epsilon_{5}\right) \times\left(E_{k}^{W}\right)^{T}\left(E_{k}^{W}\right), \\
& \Omega_{9,11}^{k}=\Omega_{9,12}^{k}=0, \Omega_{10,10}^{k}=-2 K_{3}+\left(1-\alpha_{0}\right)^{2}\left(\epsilon_{1}+\bar{\tau}_{1} \epsilon_{2}+\bar{\tau}_{2} \epsilon_{4}\right)\left(E_{k}^{W}\right)^{T}\left(E_{k}^{W}\right) \\
& +\sigma^{2}\left(\bar{\tau}_{1} \epsilon_{3}+\bar{\tau}_{2} \epsilon_{5}\right)\left(E_{k}^{W}\right)^{T}\left(E_{k}^{W}\right), \Omega_{10,11}^{k}=\Omega_{10,12}^{k}=0,
\end{aligned}
$$




$$
\Omega_{11,11}^{k}=-\left(\frac{1-\alpha_{0}}{\bar{\tau}_{1}}\right) Z_{1}, \Omega_{11,12}^{k}=0, \Omega_{12,12}^{k}=-\left(\frac{\alpha_{0}}{\bar{\tau}_{2}}\right) Z_{2} .
$$

Proof Replace $A_{k}, B_{k}, W_{k}, H_{0 k}$ and $H_{1 k}$ in LMI (16) with $A_{k}+G F(t) E_{K}^{A}$, $B_{k}+G F(t) E_{K}^{B}, W_{k}+G F(t) E_{K}^{W}, H_{0 k}+G F(t) E_{K}^{H_{0}}$ and $H_{1 k}+G F(t) E_{K}^{H_{1}}$ respectively, we find that (16) for UFSHNN (12) is equivalent to the following condition

$$
\begin{gathered}
\Upsilon_{k}+\Gamma_{1} F(t) \Pi_{1 k}^{T}+\Pi_{1 k} F^{T}(t) \Gamma_{1}^{T}+\bar{\tau}_{1} \Gamma_{2} F(t) \Pi_{1 k}^{T}+\bar{\tau}_{1} \Pi_{1 k} F^{T}(t) \Gamma_{2}^{T} \\
+\bar{\tau}_{1} \Gamma_{3} F(t) \Pi_{2 k}^{T}+\bar{\tau}_{1} \Pi_{2 k} F^{T}(t) \Gamma_{3}^{T}+\bar{\tau}_{2} \Gamma_{4} F(t) \Pi_{1 k}^{T} \\
+\bar{\tau}_{2} \Pi_{1 k} F^{T}(t) \Gamma_{4}^{T}+\bar{\tau}_{2} \Gamma_{5} F(t) \Pi_{2 k}^{T}+\bar{\tau}_{2} \Pi_{2 k} F^{T}(t) \Gamma_{5}^{T}+ \\
\Gamma_{6} F(t) \Pi_{3 k}^{T}+\Pi_{3 k} F^{T}(t) \Gamma_{6}^{T}+\Gamma_{7} F(t) \Pi_{4 k}^{T}+\Pi_{4 k} F^{T}(t) \Gamma_{7}^{T}<0
\end{gathered}
$$

where,

$$
\begin{aligned}
& \Gamma_{1}=\left[\begin{array}{llllllllllllllllllll}
G^{T} P & 0 & 0 & 0 & 0 & 0 & 0 & 0 & 0 & 0 & 0 & 0 & 0 & 0 & 0 & 0 & 0 & 0 & 0 & 0
\end{array}\right]^{T}, \\
& \Gamma_{2}=\left[\begin{array}{llllllllllllllllllll}
0 & 0 & 0 & 0 & 0 & 0 & 0 & 0 & 0 & 0 & 0 & 0 & G^{T} Z_{1} & 0 & 0 & 0 & 0 & 0 & 0 & 0
\end{array}\right]^{T} \text {, } \\
& \Gamma_{3}=\left[\begin{array}{llllllllllllllllllll}
0 & 0 & 0 & 0 & 0 & 0 & 0 & 0 & 0 & 0 & 0 & 0 & 0 & G^{T} Z_{1} & 0 & 0 & 0 & 0 & 0 & 0
\end{array}\right]^{T} \text {, } \\
& \Gamma_{4}=\left[\begin{array}{llllllllllllllllllll}
0 & 0 & 0 & 0 & 0 & 0 & 0 & 0 & 0 & 0 & 0 & 0 & 0 & 0 & G^{T} Z_{2} & 0 & 0 & 0 & 0 & 0
\end{array}\right]^{T} \text {, } \\
& \Gamma_{5}=\left[\begin{array}{lllllllllllllllllllll}
0 & 0 & 0 & 0 & 0 & 0 & 0 & 0 & 0 & 0 & 0 & 0 & 0 & 0 & 0 & G^{T} Z_{2} & 0 & 0 & 0 & 0
\end{array}\right]^{T} \text {, } \\
& \Gamma_{6}=\left[\begin{array}{llllllllllllllllllll}
0 & 0 & 0 & 0 & 0 & 0 & 0 & 0 & 0 & 0 & 0 & 0 & 0 & 0 & 0 & 0 & G^{T} \bar{P} & 0 & 0 & 0
\end{array}\right]^{T} \text {, }
\end{aligned}
$$

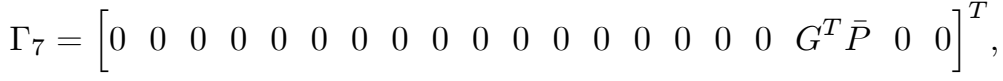

$$
\begin{aligned}
& \Pi_{1 k}=\left[\begin{array}{llllllllllllllllllll}
-E_{k}^{A} & 0 & 0 & 0 & 0 & 0 & 0 & E_{k}^{B} & \alpha_{0} E_{k}^{W} & \left(1-\alpha_{0}\right) E_{k}^{W} & 0 & 0 & 0 & 0 & 0 & 0 & 0 & 0 & 0 & 0
\end{array}\right]^{T}, \\
& \Pi_{2 k}=\left[\begin{array}{llllllllllllllllllllll}
0 & 0 & 0 & 0 & 0 & 0 & 0 & 0 & \sigma E_{k}^{W} & -\sigma E_{k}^{W} & 0 & 0 & 0 & 0 & 0 & 0 & 0 & 0 & 0 & 0
\end{array}\right]^{T}, \\
& \Pi_{3 k}=\left[\begin{array}{lllllllllllllllllll}
-E_{k}^{H_{0}} & 0 & \alpha_{0} E_{k}^{H_{1}} & 0 & \left(1-\alpha_{0}\right) E_{k}^{H_{1}} & 0 & 0 & 0 & 0 & 0 & 0 & 0 & 0 & 0 & 0 & 0 & 0 & 0 & 0
\end{array}\right]^{T} \text {, } \\
& \Pi_{4 k}=\left[\begin{array}{llllllllllllllllllll}
0 & 0 & \sigma E_{k}^{H_{1}} & 0 & \sigma E_{k}^{H_{1}} & 0 & 0 & 0 & 0 & 0 & 0 & 0 & 0 & 0 & 0 & 0 & 0 & 0 & 0 & 0
\end{array}\right]^{T} .
\end{aligned}
$$

From Lemma 2.10, (44) holds for all $F^{T}(t) F(t) \leq I$ if and only if there exist scalars $\varepsilon_{j}>0(j=1,2, \ldots, 7)$, such that

$$
\begin{gathered}
\Upsilon_{k}+\varepsilon_{1}^{-1} \Gamma_{1} \Gamma_{1}^{T}+\varepsilon_{1} \Pi_{1 k} \Pi_{1 k}^{T}+\bar{\tau}_{1} \varepsilon_{2}^{-1} \Gamma_{2} \Gamma_{2}^{T}+\bar{\tau}_{1} \varepsilon_{2} \Pi_{1 k} \Pi_{1 k}^{T}+\bar{\tau}_{1} \varepsilon_{1}^{-1} \Gamma_{3} \Gamma_{3}^{T} \\
+\bar{\tau}_{1} \varepsilon_{3} \Pi_{2 k} \Pi_{2 k}^{T}+\bar{\tau}_{2} \varepsilon_{4}^{-1} \Gamma_{4} \Gamma_{4}^{T}+\bar{\tau}_{2} \varepsilon_{4} \Pi_{1 k} \Pi_{1 k}^{T}+\bar{\tau}_{2} \varepsilon_{5}^{-1} \Gamma_{5} \Gamma_{5}^{T}+\bar{\tau}_{2} \varepsilon_{5} \Pi_{2 k} \Pi_{2 k}^{T}
\end{gathered}
$$




$$
+\varepsilon_{6}^{-1} \Gamma_{6} \Gamma_{6}^{T}+\varepsilon_{6} \Pi_{3 k} \Pi_{3 k}^{T}+\varepsilon_{7}^{-1} \Gamma_{7} \Gamma_{7}^{T}+\varepsilon_{7} \Pi_{4 k} \Pi_{4 k}^{T}<0 .
$$

By Schur complement, the Eq. (45) is equivalent to the LMI (43). Then, by Theorem 3.1, the system (12) is globally robustly asymptotically stable in the mean square. This completes the proof.

Remark 3.3 In 22], the authors dealt with the problem of delay-dependent robust stability for uncertain stochastic fuzzy Hopfield neural networks with timevarying delays. However, the probability distribution delay was not taken into account in this model. In our paper, we study delay-dependent robust stability analysis for uncertain fuzzy stochastic Hopfield neural networks with random timevarying delays. Thus, the results in this paper are lead to an improvement over the existing ones 22].

Remark 3.4 In the case of $k=1$, the system (12) is reduced to same as in 18 and the stability criteria for the corresponding reduced system can be obtained by using Theorem 3.1. Moreover, the traditional assumption such as boundedness, monotonicity or differentiability on the neuron activation functions 22 have been removed in this paper.

\section{Numerical Examples}

In this section, we will give two examples showing the effectiveness of established theoretical results.

Example 1 Consider the SNNs (14) without uncertain parameters defined as

$$
\begin{gathered}
A_{1}=\left[\begin{array}{ll}
3 & 0 \\
0 & 5
\end{array}\right], B_{1}=\left[\begin{array}{cc}
0.6 & -0.4 \\
0.5 & 0.4
\end{array}\right], W_{1}=\left[\begin{array}{cc}
0.3 & 0.4 \\
0.2 & -0.5
\end{array}\right], \\
H_{01}=\left[\begin{array}{cc}
0.7 & 0.5 \\
-0.8 & 0.5
\end{array}\right], H_{11}=\left[\begin{array}{cc}
0.1 & 0 \\
0 & 0.3
\end{array}\right], A_{2}=\left[\begin{array}{ll}
4 & 0 \\
0 & 6
\end{array}\right], \\
B_{2}=\left[\begin{array}{cc}
0.5 & -0.6 \\
0.6 & 0.5
\end{array}\right], \quad W_{2}=\left[\begin{array}{cc}
0.4 & 0.3 \\
0.2 & -0.4
\end{array}\right], \\
H_{02}=\left[\begin{array}{cc}
0.5 & -0.5 \\
0.7 & 0.5
\end{array}\right], H_{12}=\left[\begin{array}{cc}
0.1 & 0 \\
0 & 0.2
\end{array}\right], L=\left[\begin{array}{ll}
1 & 0 \\
0 & 1
\end{array}\right] .
\end{gathered}
$$

The activation function $f(x(t))=\tanh (x(t))$, the time-varying delays are chosen as $\bar{\tau}_{1}=0.4$ and $\bar{\tau}_{2}=1$. The derivative of time-varying delays $\dot{\tau}_{1}(t) \leq \mu_{1}=0.9$, $\dot{\tau}_{2}(t) \leq \mu_{2}=0.9, \alpha_{0}=0.2$, and using the Matlab LMI toolbox to solve the LMI in Theorem 3.1, we obtained the following matrices

$$
P=\left[\begin{array}{cc}
327.4076 & 24.6582 \\
24.6582 & 186.7410
\end{array}\right], Q_{1}=\left[\begin{array}{cc}
29.1065 & 2.8428 \\
2.8428 & 78.8062
\end{array}\right],
$$




$$
\begin{aligned}
& Q_{2}=\left[\begin{array}{cc}
28.3027 & 2.5709 \\
2.5709 & 73.2516
\end{array}\right], Q_{3}=\left[\begin{array}{cc}
28.3027 & 2.5709 \\
2.5709 & 73.2516
\end{array}\right], \\
& Q_{4}=\left[\begin{array}{cc}
29.1065 & 2.8428 \\
2.8428 & 78.8062
\end{array}\right], Q_{5}=\left[\begin{array}{cc}
124.1846 & 7.8651 \\
7.8651 & 200.4389
\end{array}\right], \\
& Q_{6}=\left[\begin{array}{cc}
361.9906 & 25.6513 \\
25.6513 & 445.1012
\end{array}\right], Z_{1}=\left[\begin{array}{cc}
53.0635 & 4.5276 \\
4.5276 & 19.6996
\end{array}\right], \\
& Z_{2}=\left[\begin{array}{cc}
34.4196 & 3.2734 \\
3.2734 & 10.2579
\end{array}\right], R_{1}=\left[\begin{array}{cc}
48.6955 & 15.8265 \\
15.8265 & 45.5044
\end{array}\right], \\
& R_{2}=\left[\begin{array}{cc}
27.2106 & 10.5027 \\
10.5027 & 24.3706
\end{array}\right], X=10^{5} \times\left[\begin{array}{cc}
0.0003 & 2.3024 \\
-2.3023 & 0.0007
\end{array}\right], \\
& Y=\left[\begin{array}{cc}
9.9647 & 0.3887 \\
6.4928 & -2.2659
\end{array}\right], K_{1}=\left[\begin{array}{cc}
139.1778 & 0 \\
0 & 139.1778
\end{array}\right], \\
& K_{2}=\left[\begin{array}{cc}
13.2926 & 0 \\
0 & 13.2926
\end{array}\right], K_{3}=\left[\begin{array}{cc}
33.5343 & 0 \\
0 & 33.5343
\end{array}\right] .
\end{aligned}
$$

Therefore, it follows from Theorem 3.1, that the SNNs without uncertain parameters (14) is globally asymptotically stable in the mean square. The response of the state dynamics for the SNNs without uncertain parameters (14) which converges to zero asymptotically in the mean square are shown in Figures 1 and 2.

Example 2 Consider the SNNs (12) with uncertain parameters defined as

$$
\begin{gathered}
A_{1}=\left[\begin{array}{ll}
3 & 0 \\
0 & 4
\end{array}\right], B_{1}=\left[\begin{array}{cc}
0.5 & -0.7 \\
0.3 & 0.6
\end{array}\right], W_{1}=\left[\begin{array}{cc}
0.4 & 0.3 \\
0.4 & -0.5
\end{array}\right], \\
H_{01}=\left[\begin{array}{ll}
0.5 & 0.7 \\
0.7 & 0.6
\end{array}\right], H_{11}=\left[\begin{array}{cc}
0.2 & 0 \\
0 & 0.1
\end{array}\right], A_{2}=\left[\begin{array}{ll}
3 & 0 \\
0 & 5
\end{array}\right], \\
B_{2}=\left[\begin{array}{cc}
-0.4 & -0.6 \\
0.6 & 0.4
\end{array}\right], W_{2}=\left[\begin{array}{cc}
0.2 & 0.3 \\
0.2 & -0.4
\end{array}\right], H_{02}=\left[\begin{array}{ll}
0.7 & 0.5 \\
0.6 & 0.5
\end{array}\right], \\
H_{12}=\left[\begin{array}{cc}
0.2 & 0 \\
0 & 0.1
\end{array}\right], L=\left[\begin{array}{cc}
1 & 0 \\
0 & 1
\end{array}\right], G=\left[\begin{array}{cc}
0.2 & 0 \\
0 & 0.3
\end{array}\right], \\
E_{1}^{A}=E_{2}^{A}=E_{1}^{B}=E_{2}^{B}=E_{1}^{W}=E_{2}^{W}=E_{1}^{H_{0}}=E_{2}^{H_{0}}=E_{1}^{H_{1}}=E_{2}^{H_{1}}=0.2 I .
\end{gathered}
$$

The activation function $f(x(t))=\tanh (x(t))$, the time-varying delays are chosen as $\bar{\tau}_{1}=0.2, \bar{\tau}_{2}=1.2$, The derivative of time-varying delays $\dot{\tau}_{1}(t) \leq \mu_{1}=0.8$, $\dot{\tau}_{2}(t) \leq \mu_{2}=0.8, \alpha_{0}=0.1$ and using the Matlab LMI toolbox to solve the LMI in Theorem 3.2, we obtained the following matrices

$$
P=\left[\begin{array}{cc}
518.3570 & 41.1464 \\
41.1464 & 291.9800
\end{array}\right], Q_{1}=\left[\begin{array}{cc}
19.7588 & -25.7369 \\
-25.7369 & 50.5741
\end{array}\right],
$$




$$
\begin{aligned}
& Q_{2}=\left[\begin{array}{cc}
19.4515 & -25.2105 \\
-25.2105 & 49.6365
\end{array}\right], Q_{3}=\left[\begin{array}{cc}
19.4515 & -25.2105 \\
-25.2105 & 49.6365
\end{array}\right], \\
& Q_{4}=\left[\begin{array}{cc}
13.4952 & -17.7217 \\
-17.7217 & 34.7562
\end{array}\right], Q_{5}=\left[\begin{array}{cc}
124.1216 & -9.4733 \\
-9.4733 & 95.1319
\end{array}\right] \text {, } \\
& Q_{6}=\left[\begin{array}{cc}
970.4180 & 52.1610 \\
52.1610 & 539.2814
\end{array}\right], Z_{1}=\left[\begin{array}{cc}
58.4896 & -27.6329 \\
-27.6329 & 38.2542
\end{array}\right] \text {, } \\
& Z_{2}=\left[\begin{array}{cc}
21.3870 & -12.1165 \\
-12.1165 & 12.1744
\end{array}\right], R_{1}=\left[\begin{array}{cc}
70.7114 & -67.4364 \\
-67.4364 & 83.8331
\end{array}\right] \text {, } \\
& R_{2}=\left[\begin{array}{cc}
34.0450 & -34.7188 \\
-34.7188 & 40.1826
\end{array}\right], X=\left[\begin{array}{cc}
19.1175 & -23.9969 \\
-23.9969 & 47.1209
\end{array}\right] \text {, } \\
& Y=\left[\begin{array}{cc}
6.2330 & -2.7589 \\
-2.7589 & 3.6739
\end{array}\right], K_{1}=\left[\begin{array}{cc}
345.0867 & 0 \\
0 & 345.0867
\end{array}\right] \text {, } \\
& K_{2}=\left[\begin{array}{cc}
13.8953 & 0 \\
0 & 13.8953
\end{array}\right], K_{3}=\left[\begin{array}{cc}
111.8889 & 0 \\
0 & 111.8889
\end{array}\right] \text {, } \\
& \epsilon_{1}=248.1167, \quad \epsilon_{2}=167.8423, \quad \epsilon_{3}=492.0111, \quad \epsilon_{4}=34.7926 \text {, } \\
& \epsilon_{5}=150.2244, \quad \epsilon_{6}=279.6797, \quad \epsilon_{7}=97.3928 .
\end{aligned}
$$

Therefore, it follows from Theorem 3.2, that the UFSHNN (12) is globally robustly asymptotically stable in the mean square. The response of the state dynamics for the UFSHNN (12) which converges to zero asymptotically in the mean square are shown in Figures 3 and 4.

\section{Conclusion}

The delay-dependent robust stability analysis for fuzzy stochastic Hopfield neural networks with random time-varying delays has been investigated. By using the combination of Lyapunov stability theory and stochastic analysis approach, some delay-dependent criteria have been derived to guarantee that the global robust asymptotic stability of the system in the mean square. This criteria can be checked easily by the LMI control toolbox in Matlab. Finally, numerical examples have been provided to illustrate the advantages and usefulness of the proposed results.

\section{REFERENCES}

[1] Haykin, S., Neural Networks: A Comprehensive Foundation, Prentice Hall, NJ, 1998.

[2] Arik, S., Stability analysis of delayed neural networks, IEEE Transactions on Circuits and Systems, I 47 (1997), 1089-1092.

[3] Liu, Y. R., Wang, Z. D., Liu, X., Global exponential stability of generalized recurrent neural networks with discrete and distributed delays, Neural Networks, 19 (2006), 667-675.

[4] Zhang, Q., Wei, X., Xu, J., Delay-dependent global stability condition for delayed Hopfield neural networks, Nonlinear Analysis: Real World Applications, 8 (2007), 997-1002. 


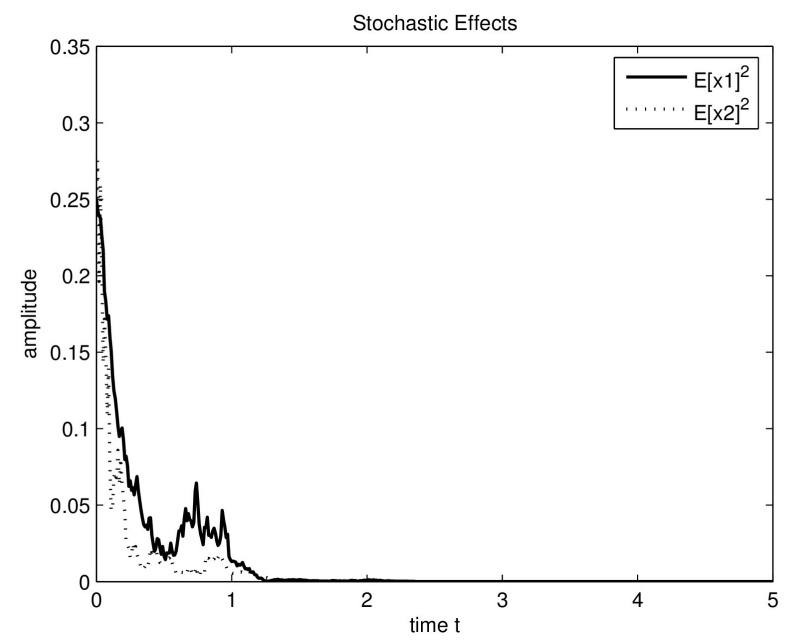

FiguRE 1. The state trajectories are converging to zero for $k=1$ for Example 1

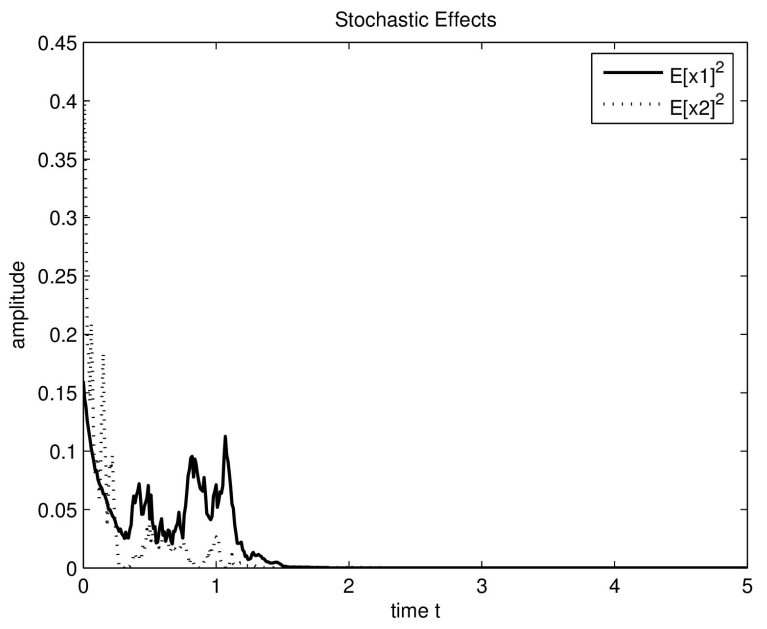

FiguRE 2. The state trajectories are converging to zero for $k=2$ for Example 1

[5] Wu, H., Feng, W., Liang, X., New stability criteria for uncertain neural networks with interval time-varying delays, Cognitive Neurodynamics, 2 (2008), 363-370.

[6] Li, X., Chen, Z., Stability properties for Hopfield neural networks with delays and impulsive perturbations, Nonlinear Analysis: Real World Applications, 10 (2009), 3253-3265. 


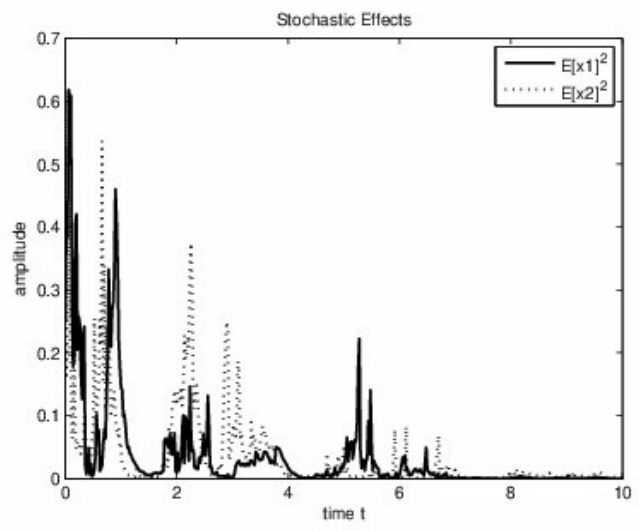

FiguRE 3. The state trajectories are converging to zero for $k=1$ for Example 2

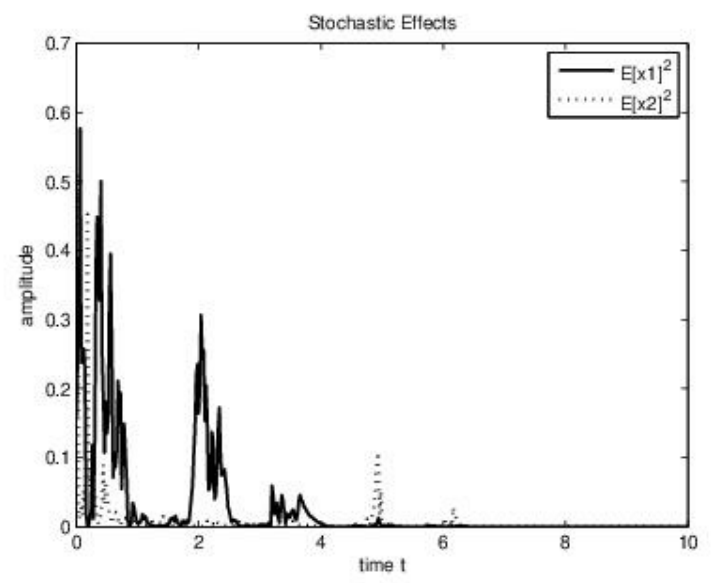

FiguRE 4. The state trajectories are converging to zero for $k=2$ for Example 2

[7] Shao, H., Novel delay-dependent stability results for neural networks with time-varying delays, Circuits Systems and Signal Processings, 29 (2010), 637-647.

[8] Chen, W.H., Lu, X., Mean square exponential stability of uncertain stochastic delayed neural networks, Physics Letters A, 372 (2008), 1061-1069.

[9] Feng, W., Yang, S. X., Wu, H., On robust stability of uncertain stochastic neural networks with distributed and interval time-varying delays, Chaos, Solitons and Fractals, 42 (2009), 2095-2104.

[10] Ma, L., Da, F., Mean-square exponential stability of stochastic Hopfield neural networks with time-varying discrete and distributed delays, Physics Letters A, 373 (2009), 2154-2161. 
[11] Lou, X., Cui, B., Delay-dependent stochastic stability of delayed Hopfield neural networks with Markovian jump parameters, Journal of Mathematical Analysis and Applications, 328 (2007), 316-326.

[12] Rakkiyappan, R., Balasubramaniam, P., Delay-dependent asymptotic stability for stochastic delayed recurrent neural networks with time varying delays, Applied Mathematics and Computation, 198 (2008), 526-533.

[13] Zhou, Q., Wan, L., Exponential stability of stochastic delayed Hopfield neural networks, Applied Mathematics and Computation, 199 (2008), 84-89.

[14] Sakthivel, R., Samidurai, R., Anthoni, S.M., Asymptotic stability of stochastic delayed recurrent neural networks with impulsive effects, Journal of Optimization Thoery and Applications, 147 (2010), 583-596.

[15] Sakthivel, R., Samidurai, R., Anthoni, S.M., New exponential stability criteria for stochastic BAM neural networks with impulses, Physica Scripta, 82 (2010), 045802.

[16] Sakthivel, R., Raja, R., Anthoni, S.M., Asymptotic stability of delayed stochastic genetic regulatory networks with impulses, Physica Scripta, 82 (2010), 055009.

[17] Zhang, Y. J., Yue, D., Tian, E. G., Robust delay-distribution-dependent stability of discretetime stochastic neural networks with time-varying delay, Neurocomputing, 72 (2009), 12651273.

[18] Fu, J., Zhang, H. G., Ma, T., Delay-probablity-distribution-dependent robust stability analysis for stochastic neural networks with time-varying delays, Progress Natural Science, 19 (2009), 1333-1340.

[19] Takagi, T., Sugeno, M., Fuzzy identification of systems and its applications to modeling and control, IEEE Transactions on System, Man, and Cybernetics, 15 (1985), 116-132.

[20] Huang, H., Ho, D.W.C., Lam, J., Stochastic stability analysis of fuzzy Hopfield neural networks with time-varying delays, IEEE Transactions on Circuits and Systems, II 52 (2005) 251-255.

[21] Li, H., Chen, B., Lin, C., Zhou, Q., Mean square exponential stability of stochastic fuzzy Hopfield neural networks with discrete and distributed time-varying delays, Neurocomputing, 72 (2009) 2017-2023.

[22] Sheng, L., Gao, M., Yang, H., Delay-dependent robust stability for uncertain stochastic fuzzy Hopfield neural networks with time-varying delays, Fuzzy Sets and Systems, 160 (2009), 35033517 .

[23] Rakkiyappan, R., Balasubramaniam, P., Delay-probability-distribution-dependent stability of uncertain stochastic genetic regulatory networks with mixed time-varying delays: An LMI approach, Nonlinear Analysis: Hybrid Systems, 4 (2010), 600-607.

[24] Gu, K., Integral inequality in the stability problem of time-delay Systems, Proceeding 39th IEEE CDC, Sydney, Philadelphia, 1994.

[25] Boyd, S., Ghaoui, L. E., Feron, E., Balakrishnan, V., Linear Matrix Inequalities in Systems and Control Theory, SIAM books, Philadelphia, 1994.

[26] Xie, L., Output feedback $H_{\infty}$ control of systems with parameter uncertainty, International Journal of Control, 63(1996)741-750.

[27] Moon, Y.S., Park, P., Kwon, W.H., Lee, Y.S., Delay-dependent robust stabilization of uncertain state-delayed systems, International Journal of Control, 74 (2001), 1447-1455. 